\title{
Aktivierende Erwerbsminderungsrente? Zur Rückkehr auf den Arbeitsmarkt nach Erwerbsminderung
}

\author{
Martin Brussig • Susanne Eva Drescher • Thorsten Kalina
}

Online publiziert: 20. April 2020

(C) Der/die Autor(en) 2020

Zusammenfassung Die Sicherung gegen Erwerbsminderung gehört zu den klassischen Aufgaben des Sozialstaates. Im deutschen Rentenrecht sind Erwerbsminderungsrenten grundsätzlich zu befristen. Darin drückt sich die Annahme aus, dass eine Erwerbsminderung im Regelfall nur vorübergehend währen würde. Durch die Reform des Erwerbsminderungsrechts 2001 wurde das Ziel der Rückkehr in Beschäftigung gestärkt. Der Beitrag untersucht, wie oft vor Vollendung des 60. Lebensjahres nach einer Erwerbsminderungsrente der Übergang in Beschäftigung gelingt und wem sie gelingt. Die Untersuchungsergebnisse auf Basis der Versichertenkontenstichprobe 2015 zeigen, dass 12,6\% der ehemaligen Erwerbsminderungsrentner_innen zwölf Monate nach dem Auslaufen der Rente wieder in Beschäftigung sind; weitere 8,7\% sind arbeitslos. Der überwiegende Anteil scheidet mit Vollendung des 60. Lebensjahres aus der Beobachtung aus; hier ist letztlich ein dauerhafter Rentenbezug sehr wahrscheinlich. Vor allem Jüngeren gelingt der Übergang in einen erwerbsnahen Zustand (beschäftigt oder arbeitslos). Auch nach dem Ende einer Erwerbsminderungsrente sind die Erwerbsverläufe oft instabil. Eine Wirkung aufgrund der Reform von 2001 ist nicht zu erkennen.

Schlüsselwörter Erwerbsminderung $\cdot$ Erwerbsminderungsrente $\cdot$ Aktivierende Sozialpolitik $\cdot$ Erwerbsintegration

\footnotetext{
M. Brussig $(\bowtie) \cdot$ S. E. Drescher · T. Kalina Institut Arbeit und Qualifikation (IAQ), Universität Duisburg-Essen, 47048 Duisburg, Deutschland E-Mail: martin.brussig@uni-due.de

S. E. Drescher

E-Mail: susanne.eva.drescher@uni-due.de

T. Kalina

E-Mail: thorsten.kalina@uni-due.de
} 


\title{
Reduced earning capacity pensions as an instrument for activation? Return to work after a reduction in earning capacity
}

\begin{abstract}
Protection from reduced earning capacity is one of the classic tasks of the welfare state. Under German pension law, reduced earning capacity pensions are generally limited in time. This reflects the assumption that a reduction in earning capacity would normally only be temporary. The reform of the system in 2001 strengthened the statutory goal of return to work. This article examines how often the transition from disability benefits to employment is successful before the age of 60 and for whom. The results based on social security data show that 12.6 per cent of formerly incapacitated persons are back in employment within twelve months after the pension expires; a further 8.7 per cent are unemployed. The overwhelming majority drops out of observation when they reach the age of 60; in this case, it is highly probable that they will receive a permanent pension. Younger people, in particular, are successful in transitioning to a situation close to employment (employed or unemployed). Even after the end of reduced earning capacity benefits, employment histories remain often unstable. An effect of the 2001 reform is not discernible.
\end{abstract}

Keywords Reduced earning capacity - Reduced earning capacity benefits · Activating social policy $\cdot$ Integration into employment

\section{Une pension d'invalidité activatrice ? À propos du retour sur le marché du travail après une période d'invalidité}

Résumé L'assurance invalidité fait partie des missions classiques de l'État-providence. En droit allemand, les pensions d'invalidité sont en principe accordées pour une durée limitée, toute réduction de la capacité de travail étant de manière générale réputée temporaire. La réforme du droit des pensions d'invalidité de 2001 a renforcé l'objectif d'un retour à l'emploi. Cet article étudie le taux de retour à l'emploi après une période d'invalidité chez les personnes de moins de 60 ans ainsi que le profil de ceux qui y parviennent. Les résultats de l'enquête conduite sur la base de l'échantillon des assurés de 2015 montrent que 12,6 pourcent des anciens bénéficiaires d'une pension d'invalidité ont retrouvé un emploi douze mois après l'expiration de leur pension tandis que 8,7 pourcent sont au chômage. La grande majorité d'entre eux disparaissent de la population observée quand ils atteignent l'âge de 60 ans, auquel cas il est en définitive très probable qu'ils perçoivent une pension à titre permanent. Ce sont surtout les plus jeunes qui réussissent le retour à la vie active (emploi ou chômage). Même après la fin de la période d'invalidité, les parcours professionnels sont souvent instables. Un effet de la réforme de 2001 n'est pas à constater.

Mots-clés Invalidité · Pension d'invalidité · Politique sociale d'activation · Insertion professionnelle 


\section{Einleitung}

Die klassischen Sozialversicherungen federn Risiken der Arbeitslosigkeit, bei Krankheit, des Alters und bei Erwerbsunfähigkeit ab. Damit sie ihren Schutz entfalten können, muss die Struktur sozialer Risiken den Regelungen zur sozialen Sicherung entsprechen. Hierfür ist die Rentenversicherung ein gutes Beispiel. Zum Zeitpunkt ihrer Einführung in Deutschland im Jahr 1889 galt für den Bezug einer Altersrente eine Altersgrenze von 70 Jahren, die den Zeitgenossen und mehr noch den Nachgeborenen im Rückblick als überhöht und damit als faktisch unwirksam erschien. Doch zeitgleich mit der Altersrente wurde die Invalidenrente eingeführt. Sie sicherte das Risiko der Erwerbsminderung (in zeitgenössischer Diktion: der Invalidität) unabhängig vom Alter. Auch heute noch ist die Erwerbsminderungsrente ein zentraler Bestandteil im System der sozialen Sicherung. Sie sichert die materielle Existenz, wenn Menschen im erwerbsfähigen Alter aus gesundheitlichen Gründen dauerhaft nicht in der Lage sind, ihre Kräfte und Fähigkeiten für eine Erwerbstätigkeit einzusetzen.

Das Erwerbsminderungsrentenrecht wurde 2001 reformiert. Diese Reform war die bis dahin (und seitdem) einschneidendste Veränderung seit der Einführung der Rentenversicherung 1889 bzw. seit Einführung der Versicherung gegen Berufsunfähigkeit 1913. Die Reform des Erwerbsminderungsrentenrechts stand im Einklang mit weiteren aktivierenden Reformen in der Arbeitsmarkt- und Sozialpolitik in Deutschland und darüber hinaus (Fay 1996; OECD 1996; Martin 1998) seit den 1990erJahren. Als ,aktivierend“ werden Sozialpolitiken bezeichnet, die darauf orientieren, nichterwerbstätige Sozialleistungsbeziehende in Beschäftigung zu integrieren und sie zu erwerbstätigen Steuer- und Beitragszahlenden zu machen. Der Erwerbsarbeit wird ein starker Vorrang vor dem Bezug von Sozialleistungen gegeben. Aktivierende Sozialpolitiken arbeiten mit Anreizen und Sanktionen, oftmals auch mit Leistungskürzungen in den Sozialsystemen, und lassen dadurch den Sozialleistungsbezug weniger attraktiv erscheinen. Maßgeblich hierfür ist die Vorstellung, dass Personen sich zwischen Erwerbstätigkeit und Sozialleistungsbezug entscheiden können und deshalb die Anreize für eine Erwerbstätigkeit verstärkt bzw. für einen Sozialleistungsbezug geschwächt werden müssen. Aktivierende Sozialpolitiken bieten aber auch verschiedene Formen der Unterstützung bei der Suche und Aufnahme einer Beschäftigung (Brettschneider 2007; Lessenich 2008; Aurich 2011).

Im vorliegenden Aufsatz werden die Auswirkungen der reformierten Erwerbsminderungsrente anhand eines signifikanten Reformbausteins - der grundsätzlichen Befristung des Rentenbezuges - untersucht. Die regelhafte Befristung einer Erwerbsminderungsrente im gegenwärtigen Recht impliziert die Annahme, dass Erwerbsminderungen normalerweise nur vorübergehend seien, danach die Erwerbsfähigkeit wieder hergestellt wäre und eine Erwerbsintegration wieder erreicht werden würde. Es ist jedoch nicht bekannt, wie oft nach dem Auslaufen einer Erwerbsminderungsrente die Rückkehr in Erwerbstätigkeit gelingt. In diesem Aufsatz werden deshalb die individuellen Beschäftigungschancen nach einer befristeten Erwerbsminderungsrente untersucht, um einen Beitrag zum Verständnis der sozialen Sicherung bei Erwerbsminderung zu leisten. Darüber hinaus bietet die Analyse wichtige Informationen, um die Angemessenheit der gegenwärtigen Absicherung des Erwerbs- 
minderungsrentenrisikos zu beurteilen und Anstöße zu ihrer Weiterentwicklung zu geben.

Im folgenden Abschnitt des Beitrags werden die Regelungen im Erwerbsminderungsrentenrecht erläutert und eingeordnet (Abschn. 2). Es wird herausgearbeitet, dass die grundsätzliche Befristung der Erwerbsminderungsrente sich als ein Element der aktivierenden Sozialpolitik verstehen lässt und erst im Zuge der aktivierenden Sozialrechtsreformen vom Ausnahmefall zum Regelfall umdefiniert wurde. Daran schließt sich ein Bericht zum Forschungsstand über Erwerbsverläufe nach dem Auslaufen einer befristeten Erwerbsminderungsrente an (Abschn. 3). Hierbei wird deutlich, dass sich die bisherige Forschung überwiegend mit dem Zugang in Erwerbsminderungsrente beschäftigt hat, nicht aber mit dem sozialpolitisch ebenso bedeutsamen Ende der Erwerbsminderung. Die empirischen Analysen beginnen mit einer Präsentation der verwendeten Daten, bevor Ergebnisse über den Verbleib nach einer Erwerbsminderungsrente vorgestellt werden (Abschn. 4 und 5). Die Auswertungen werden entlang von zwei Hypothesenkomplexen präsentiert, nämlich der Differenzierung entlang von soziodemografischen Merkmalen und der Differenzierung entlang von berufsbiografischen Merkmalen. Abschließend werden die Ergebnisse eingeordnet und Schlussfolgerungen aus den vorgestellten Befunden gezogen (Abschn. 6).

\section{Die Regelungen im Erwerbsminderungsrentenrecht und ihre sozialpolitische Einordnung}

\subsection{Die rechtlichen Regelungen: Betonung der individuellen Leistungsfähigkeit}

Um eine Erwerbsminderungsrente $\mathrm{zu}$ erhalten, sind persönliche und versicherungsrechtliche Voraussetzungen zu erfüllen. Die persönliche Voraussetzung ist, dass eine Erwerbsminderung vorliegt. Die versicherungsrechtlichen Voraussetzungen regeln, wann eine Rente bezogen werden kann. Die wichtigste versicherungsrechtliche Voraussetzung ist, dass in den fünf Jahren vor Eintritt der Erwerbsminderung in mindestens 36 Monaten Beiträge an die Rentenversicherung - im Regelfall aus versicherungspflichtiger Beschäftigung - entrichtet wurden. Durch diese Bedingung, die Mitte der 1980er-Jahre eingeführt wurde und den bis heute schärfsten Einschnitt bei den Neuzugängen in Erwerbsminderungsrenten verursachte, wurde geregelt, dass nur solches Arbeitsvermögen gegen das Risiko der Erwerbsminderung versichert ist, das zuvor auch tatsächlich für Erwerbszwecke eingesetzt wurde. ${ }^{1}$

Die Erwerbsfähigkeit wird als Fähigkeit bestimmt, eine bestimmte Zahl von Stunden pro Tag an fünf Tagen in der Woche unter den ,üblichen Bedingungen des allgemeinen Arbeitsmarktes“ (§ 43 SGB VI) erwerbstätig sein zu können. Bei einer Erwerbsfähigkeit von unter drei Stunden pro Tag liegt eine volle Erwerbsminderung

\footnotetext{
1 Darüber hinaus gibt es eine „lange Wartezeit“ von 20 Jahren. Eine Erwerbsminderungsrente wird auch bewilligt, wenn eine Erwerbsminderung vor Erfüllung der allgemeinen Wartezeit vorlag und für 20 Jahre ununterbrochen bestand ( $\$ 43$ Abs. 6 SGB VI). Diese Regelung zielt auf Personen, die schon im Kinderund Jugendalter so beeinträchtigt waren, dass sie als Erwachsene nie erwerbsfähig waren.
} 
vor, bei einer Erwerbsfähigkeit von drei bis unter sechs Stunden liegt eine teilweise Erwerbsminderung vor. Insofern handelt es sich bei der Erwerbsminderung nicht um ein dichotomes, sondern um ein graduelles Konzept, wenngleich nur drei Abstufungen vorgesehen sind (keine, volle und teilweise Erwerbsminderung). Der Grad der Erwerbsfähigkeit wird durch die Rentenversicherung auf der Grundlage sozialmedizinischer Gutachten festgestellt (Stone 1985; Aurich-Beerheide et al. 2018).

Nicht im Gesetz, sondern erst durch die Rechtsprechung und die Verwaltungspraxis sind die Begriffe der ,,üblichen Bedingungen“ und des ,,allgemeinen Arbeitsmarktes" definiert. Der ,,allgemeine Arbeitsmarkt" ist in Abgrenzung vom besonderen Arbeitsmarkt in Werkstätten für behinderte Menschen sowie öffentlich geförderten Beschäftigungsprogrammen zu sehen und entspricht damit dem Arbeitsmarkt schlechthin. Sämtliche, auch einfachste Tätigkeiten kommen in Betracht, wenn die individuelle Erwerbsfähigkeit betrachtet wird. Der Begriff der ,üblichen Bedingungen" zielt auf die Anforderungen der Arbeitswelt, insbesondere die Lage, Dauer und Verteilung der Arbeitszeit. Als ,üblich“ gelten die Arbeitsbedingungen dann, wenn sie in mindestens 300 Arbeitsplätzen im Bundesgebiet vorliegen (Bundessozialgericht 2012, Rd. Nr. 18). Mit diesem extrem niedrigen Schwellenwert dürfte es praktisch nahezu unmöglich sein, ,unübliche“ Arbeitsbedingungen zu benennen, die einer Erwerbstätigkeit entgegenstehen. Ob derartige Arbeitsplätze aber für eine leistungsgeminderte Person tatsächlich erreichbar sind - und erst recht die Frage, ob diese Person für einen solchen Arbeitsplatz auch eingestellt werden würde -, spielt für die Beurteilung der individuellen Erwerbsfähigkeit keine Rolle.

Diese Regelungen, die die Erwerbsfähigkeit ausschließlich anhand der individuellen Leistungsfähigkeit beurteilen, werden als ,abstrakte Betrachtungsweise“ bezeichnet. Dem liegt die Überlegung zugrunde, dass sich das Leistungsvermögen ,abstrakt“ in Erwerbstätigkeit umsetzen lässt. Tatsächlich ist hierfür aber ein konkreter Arbeitsplatz bzw. ein konkretes Beschäftigungsverhältnis erforderlich (,,konkrete Betrachtungsweise“). Doch die Überlegung, ob tatsächlich konkrete Möglichkeiten bestehen, eine gegebene Leistungsfähigkeit für Erwerbszwecke zu verwerten, fließt nicht in die Beurteilung der Erwerbsfähigkeit ein. ${ }^{2}$

Erwerbsminderungsrenten sind im Regelfall befristet zu bewilligen ( 1102 Abs. 2 SGB VI). Als maximale Dauer der Befristung gelten drei Jahre, nach deren Ablauf die Erwerbsfähigkeit erneut geprüft wird. Erst wenn am Ende einer zweimal erneuerten Befristung die Erwerbsminderung immer noch besteht (also nach neun Jahren), geht der Gesetzgeber von einer dauerhaften Erwerbsminderung aus, und die Rente ist auf Dauer zu gewähren. Erwerbsminderungsrenten aufgrund eines verschlossenen Arbeitsmarktes sind ausschließlich wiederholt zu befristen.

\footnotetext{
2 Die konkreten Erwerbschancen fließen aber in die Beurteilung des Rentenanspruchs ein: Teilweise erwerbsgeminderte Personen haben Anspruch auf eine volle Erwerbsminderungsrente, wenn sie ihr noch vorhandenes Leistungsvermögen aufgrund der Lage am Arbeitsmarkt nicht einsetzen können. Dies wird als ,,verschlossener Arbeitsmarkt“ bezeichnet. Hiervon wird im Regelfall bei teilweise Erwerbsgeminderten ausgegangen, die langzeitarbeitslos sind. Sie erhalten eine volle Erwerbsminderungsrente (BSG, Beschlüsse vom 10.12.1976 - GS 2/75, GS 3/75, GS 4/75, GS 3/76, BSGE 43, 75 ff., zitiert nach Fuchsloch 2011, S. 140).
} 
Erwerbsminderungsrenten enden mit dem Erreichen der Altersgrenze für den Renteneintritt. Sie werden dann - in der Leistungshöhe im Regelfall unverändert in Altersrenten umgewandelt ( $\$ 89$ Abs. 1 SGB VI).

Wesentliche der bis hier skizzierten rechtlichen Regelungen sind durch die Reform der Erwerbsminderungsrenten im Jahr 2001 in Kraft gesetzt worden. Versicherte, die bis zum 01.01.1961 geboren wurden, zum Zeitpunkt der Reform also das 40. Lebensjahr vollendet hatten, unterliegen dem alten Recht.

Im alten Recht wurde die Erwerbsfähigkeit nicht anhand der Drei- und SechsStunden-Grenze beurteilt, sondern anhand einer ,,vollschichtigen“ und ,halbschichtigen“" Arbeitszeit. Systematisch gravierender ist die Abschaffung des Berufsschutzes durch die Reform: Nach dem alten Recht wurde nicht nur die Erwerbsfähigkeit, sondern auch die „Berufsunfähigkeit“ beurteilt und hierfür die erworbene Qualifikation, die überwiegend ausgeübte Tätigkeit und das erzielte Einkommen zugrunde gelegt. Man konnte berufsunfähig sein, ohne zugleich erwerbsunfähig zu sein, und erhielt dann eine Berufsunfähigkeitsrente. Eine dritte wesentliche Änderung war, dass der Befristungsgrundsatz durch die Reform umgekehrt wurde. Im alten Recht waren die Renten grundsätzlich Dauerrenten und nur zu befristen, wenn eine Besserung absehbar war, während sie im neuen Recht grundsätzlich befristet sind.

Ein Vergleich zwischen dem alten und neuen Recht der Erwerbsminderung verdeutlicht die sozialpolitische Intention der Reform, die sich als Teil der aktivierenden Sozialpolitik verstehen lässt.

\subsection{Sozialpolitische Einordnung}

Anders als bei der Funktionsweise und beim Sicherungsniveau in der Alterssicherung und im Unterschied zur Anhebung der Altersgrenzen für Altersrenten, die in der Bevölkerung außerordentlich kritisch bewertet werden (Nüchter et al. 2008; Schrenker 2009; Heinrich et al. 2016; Prinzen 2016), war die öffentliche Resonanz auf die Reform der Erwerbsminderungsrenten schwach (Brettschneider und Klammer 2016). Für eine sozialpolitische Einordnung kann daher kaum auf öffentliche Debatten zurückgegriffen werden. Es liegt daher nahe, zur Interpretation der Reform die Begründung des Gesetzgebers hinzuzuziehen (siehe Fraktionen der SPD und Bündnis 90/Die Grünen 2000). ${ }^{3}$ Doch auch dort sind nur wenige Anhaltspunkte zu den konkreten Intentionen des Gesetzgebers zu finden. Dies gilt insbesondere für die Umkehrung der Befristungsregelung mit der Befristung als Regelfall, die im Gesetzentwurf nicht begründet wurde. ${ }^{4}$

\footnotetext{
3 Zwar wurde die Reform der Erwerbsminderungsrenten im Jahr 2000 von SPD und Bündnis 90/Die Grünen verabschiedet und 2001 in Kraft gesetzt. Sie ist aber nahezu identisch mit der Reform, die 1998 von der alten Koalition von CDU/CSU und FDP angestrengt wurde, aber im Bundesrat scheiterte. Insofern kann man sagen, dass die Reform des Erwerbsminderungsrentenrechts von allen damals im Bundestag vertretenen Parteien mit Ausnahme der Linkspartei befürwortet wurde.

4 Dabei wäre dieser Reformbaustein in besonderem Maße erklärungsbedürftig, werden damit doch scheinbar widersprüchliche Anforderungen an die Rentenversicherung gestellt, die über Rentenanträge entscheiden soll. Denn maßgeblich für eine Erwerbsminderung ist nicht nur, dass aktuell die individuelle Leistungsfähigkeit unter drei bzw. sechs Stunden liegt, sondern dass sie zudem ,,auf nicht absehbare Zeit“ besteht ( $\$ 43$ SGB VI). Eine grundsätzliche Befristung erscheint widersprüchlich, da nur dauerhafte Leistungseinschränkungen eine Erwerbsminderung darstellen. Verwaltungspraktisch wird dies gelöst, indem
} 
Dennoch lässt sich auch ohne eine detaillierte Begründung des Gesetzgebers zur grundsätzlichen Befristung von Erwerbsminderungsrenten erkennen, dass mit der Reform des Erwerbsminderungsrechts der für die aktivierende Arbeitsmarkt- und Sozialpolitik charakteristische Grundsatz deutlich verstärkt wurde, der Erwerbstätigkeit den Vorrang vor dem Bezug einer Sozialleistung wie der Erwerbsminderungsrente zu geben. Vor diesem Hintergrund lässt sich die Absicht vermuten, die Leistungsdauer für Risiken zu begrenzen, die nur vorübergehend bestehen. Aber auch wenn überwiegend dauerhafte Erwerbsminderungen angenommen werden würden, kann die Entscheidung für eine grundsätzliche Befristung im Zusammenhang mit der Aktivierungspolitik begründet werden. Denn ohne Befristung der Erwerbsminderungsrente gäbe es keine Handhabe, auch für die (wenigen) Fälle, bei denen es zu einer gesundheitlichen Verbesserung kommt, eine Beschäftigungsaufnahme anzustreben.

Für die Einordnung der Reform des Erwerbsminderungsrentenrechts als aktivierende Arbeitsmarkt- und Sozialpolitik spricht zudem eine Verschärfung der Maßstäbe, anhand derer eine Erwerbsminderung festzustellen ist. Erst mit dem 2001 endgültig vollzogenen Umstieg auf die alleinige Betrachtung der regelmäßig leistbaren Arbeitsstunden erfolgte eine vollständige Abkehr vom bisherigen Recht, das in der Berücksichtigung des noch erzielbaren Einkommens für die Beurteilung der Erwerbsfähigkeit (eingeführt 1889) und des erlernten und bis zur Erwerbsminderung ausgeübten Berufes (eingeführt 1913) wurzelte.

Diese Veränderung hat die persönlichen Voraussetzungen für eine Erwerbsminderung insofern ,universalisiert“, als statusrelevante Merkmale (erlernter und ausgeübter Beruf, bislang erreichtes und mit der Leistungsminderung noch erreichbares Einkommen) für die Beurteilung der Erwerbsfähigkeit keine Rolle mehr spielen. Dementsprechend wurde der Gradualismus der Erwerbsminderung reduziert, denn seit der Reform gibt es nur noch drei Stufen der Erwerbsminderung (keine, teilweise und volle Erwerbsminderung) statt der vier Stufen vor der Reform (keine, vollschichtige und halbschichtige Erwerbsunfähigkeit sowie Berufsunfähigkeit, wobei Letztere wiederum nicht eine generelle Stufe der Leistungsminderung war, sondern im Prinzip für jeden Beruf gesondert bestimmt wurde).

Zudem wurden Leistungen gekürzt, denn die Berufsunfähigkeitsrente wurde als 2/3-Teilrente einer vollen Erwerbsunfähigkeitsrente gezahlt; die Rente wegen teilweiser Erwerbsminderung ist eine halbe Rente. Der Vorrang von Erwerbstätigkeit vor dem Bezug von Sozialleistungen ist ein zentrales Motiv der aktivierenden Arbeitsmarkt- und Sozialpolitik, und die Höhe von Sozialleistungen wird oft als Anreiz zur Rückkehr in Erwerbstätigkeit bzw. zum Verbleib in Sozialleistungen interpretiert. Leistungskürzungen setzen demnach also Anreize zur Aufnahme einer Erwerbstätigkeit (OECD 2003, S. 113 ff.). Freilich ist für den Fall der Erwerbsminderung stärker als etwa für den vorzeitigen Bezug einer Altersrente - umstritten, ob die Erwerbsminderung Ergebnis einer individuellen Wahlentscheidung ist (Bäcker 2012). Nicht zuletzt wurde der Bezug zur Erwerbstätigkeit durch den Grundsatz der Be-

keine Prognose gefordert wird, welche die gesamte Zukunft in den Blick nimmt, sondern lediglich eine für die nächsten sechs Monate (Deutsche Rentenversicherung 2014; zu § 43 SGB VI, R2.2.). Zeichnet sich für diesen Zeitraum keine Besserung ab, dann besteht die Leistungsminderung ,,auf nicht absehbare Dauer“. 


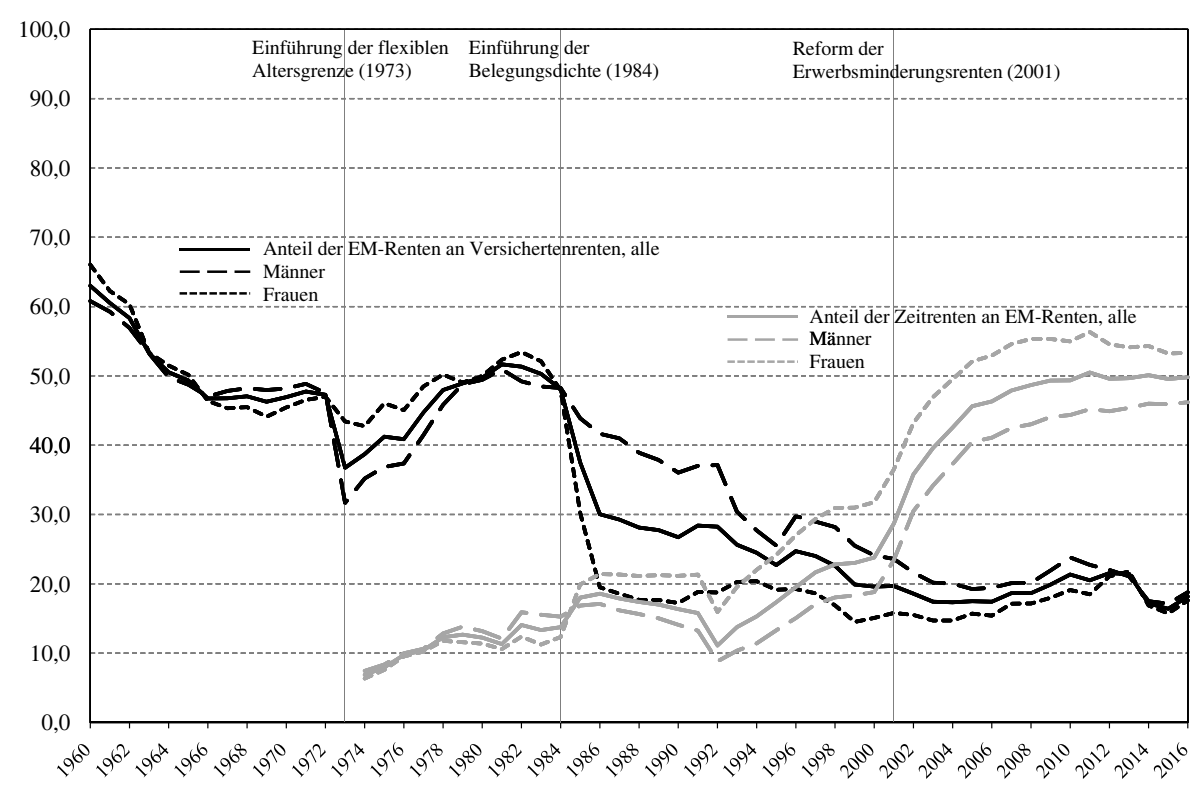

Abb. 1 Anteil von Erwerbsminderungsrenten an Versichertenrenten und Anteil von Zeitrenten an Erwerbsminderungsrenten, nach Geschlecht, 1960-2016 (ohne Knappschaftsleistungen) (in Prozent). (Quelle: Rentenversicherung in Zeitreihen, eigene Berechnungen)

fristung und damit durch die Umkehrung der bis zur Reform geltenden Regelung verstärkt.

Mit der Reform der Erwerbsminderungsrenten 2001 wurde außerdem der Grundsatz gestärkt, dass Leistungen der Rehabilitation zur Wiederherstellung der Erwerbsfähigkeit Vorrang vor Rentenleistungen haben sollen (,Reha vor Rente“). Auch dieser Baustein fügt sich in die Interpretation der reformierten Erwerbsminderungsrente als Bestandteil einer aktivierenden Sozialpolitik, soll doch damit der Bezug der Erwerbsminderungsrente zugunsten einer Integration in Beschäftigung abgewendet werden. Eine Rehabilitation während der Rente war bzw. ist hingegen weder im alten noch im neuen Recht vorgesehen, was insbesondere angesichts der grundsätzlichen Befristung der Erwerbsminderungsrente im neuen Recht und der damit verbundenen Annahme, dass Erwerbsminderungen nur vorübergehend bestehen, eine Sicherungslücke darstellt.

\subsection{Langfristige Trends zur Inanspruchnahme von Erwerbsminderungsrenten}

Aus den Daten zur Inanspruchnahme von Erwerbsminderungsrenten lassen sich Trends ebenso erkennen wie wichtige Einschnitte (siehe Abb. 1). Noch Anfang der 1960er-Jahre machten Erwerbsminderungsrenten (damals Erwerbsunfähigkeitsbzw. Berufsunfähigkeitsrenten) etwa zwei Drittel aller Neuzugänge in Versichertenrenten aus; nur etwa ein Drittel entfiel auf Altersrenten. Dies zeigt, dass eine nachberufliche Lebensphase erst in der zweiten Hälfte der 20. Jahrhunderts in Deutschland zu einer erwartbaren Normalität geworden ist; bis dahin war der Verlust der Er- 
werbsfähigkeit aufgrund von Invalidität der Regelfall und nicht die Entlassung aus der Erwerbsverpflichtung.

Weder die deutsche Einigung noch die Reform des Erwerbsminderungsrentenrechts 2001 haben deutliche Spuren in der Zeitreihe hinterlassen; die Reform sicherlich auch deshalb nicht, weil von der Neuregelung der Erwerbsminderungsrenten nur Kohorten betroffen waren, die noch nicht 40 Jahre alt und damit nicht in dem Alter waren, in dem Erwerbsminderungsrenten häufig beantragt und bewilligt werden. Einschnitte in der Inanspruchnahme der Erwerbsminderungsrente markieren vielmehr die Einführung der flexiblen Altersgrenze 1973 und die Einführung der „Belegungsdichte“ (36 Monate Versicherungszeiten in den letzten fünf Jahren vor Eintritt der Erwerbsminderung) im Jahr 1984, durch die entweder die Inanspruchnahme einer Altersrente erleichtert oder direkt der Zugang in die Erwerbsminderungsrente erschwert wurde (Schmähl 2011). Seit etwa zehn Jahren, und damit am Ende des aktuellen Beobachtungsfensters, ist eine relative Stabilität bei der Inanspruchnahme von Erwerbsminderungsrenten zu beobachten: Etwa jeder fünfte Neuzugang in eine Versichertenrente erfolgt über die Erwerbsminderungsrente.

Abb. 1 enthält außerdem den Anteil der befristeten Renten (Zeitrenten) an allen Erwerbsminderungsrenten. Ihr Anteil ist seit ihrer Einführung 1974 gestiegen und hat in den 1990er-Jahren etwa $20 \%$ erreicht. Seit der Reform des Erwerbsminderungsrentenrechts 2001 hat sich ihr Anteil mehr als verdoppelt; inzwischen ist jede zweite neu bewilligte Erwerbsminderungsrente befristet.

Die Bezugszeiten von Leistungen (Rentendauern) der Neuzugänge in Erwerbsminderungsrenten liegen mit ca. sechs Jahren (jeweils Männer und Frauen) deutlich unter den durchschnittlichen Rentenbezugszeiten der Altersrentner_innen (ca. 18 Jahre für Männer und 21 Jahre für Frauen). Bereits hier ist erkennbar, dass bei Erwerbsminderung ein vorübergehender - oft nur wenige Jahre umfassender - Rentenbezug verbreitet ist. Seit 2001 ist die mittlere Rentenbezugsdauer der Beziehenden von Erwerbsminderungsrenten sowohl bei Männern als auch bei Frauen um ca. ein Jahr gestiegen (Statistiken der Deutschen Rentenversicherung - Forschungsportal, siehe Abb. 2). Mit steigendem Alter bei Beginn der Erwerbsminderung steigen auch die durchschnittlichen Rentenbezugszeiten. Aber selbst bei den ältesten Neuzugängen in Erwerbsminderungsrente, den 64-Jährigen, liegen sie immer noch deutlich unter dem Durchschnitt der Altersrentner_innen. Lohse und Rodriguez Gonzalez (2015) zeigen, dass ein Erwerbsminderungsrentenbezug nach etwa zehn Jahren etwa ebenso oft durch Tod beendet wurde wie aus anderen Gründen. Bei Jüngeren sowie Personen mit Reha-Maßnahmen während des Rentenbezuges ist das Verhältnis zugunsten der Rückkehr in Beschäftigung günstiger (ebd.; siehe auch Rodriguez Gonzales et al. 2015).

\section{Stand des Wissens zur Rückkehr in Beschäftigung nach befristeter Erwerbsminderungsrente}

Internationale Vergleiche zeigen, dass die institutionellen Voraussetzungen zur Integration von langfristig gesundheitlich Beeinträchtigen in Beschäftigung in Deutschland relativ gut ausgebildet sind (OECD 2010, S. $101 \mathrm{f}$.): Auf einer Skala, mit 


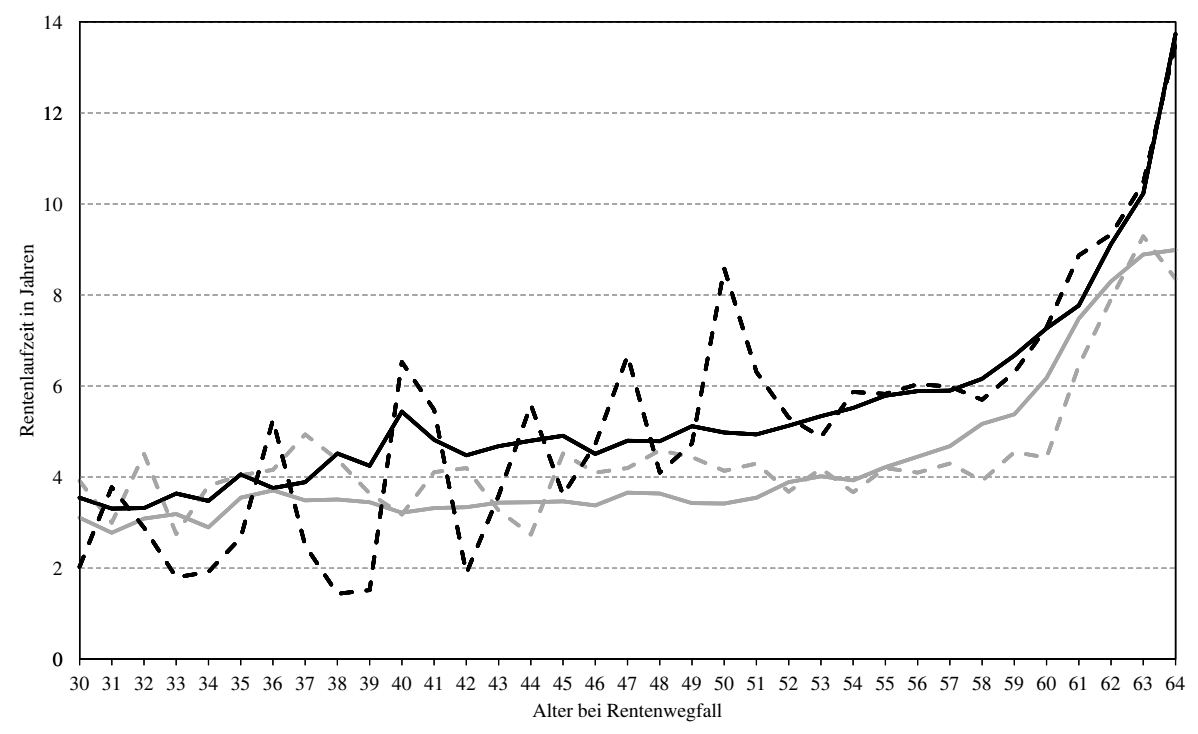

— Männer 2001 volle EM ～- - Männer 2001 teilweise EM — Männer 2014 volle EM — — Männer 2014 teilweise EM

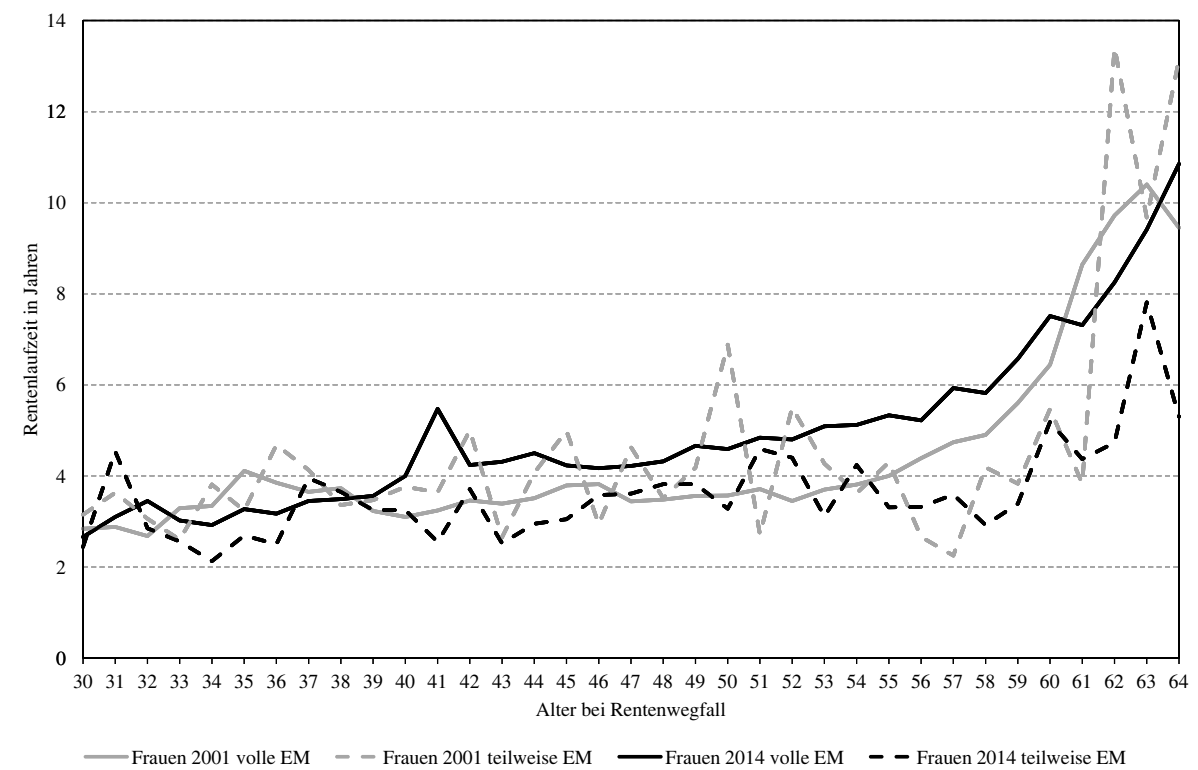

Abb. 2 Rentenbezugszeiten bei Erwerbsminderungsrenten, Männer und Frauen, 2001 und 2014. (Quelle: Statistikportal der Deutschen Rentenversicherung; Abruf am 30.11.2018) 
der Integrationspolitiken bewertet werden, erreicht Deutschland 35 Punkte (OECDDurchschnitt 24,9) und wird nur von Dänemark und Norwegen übertroffen (jeweils 37 Punkte). Diese vergleichsweise gute Position in der Bewertung durch die OECD verdankt Deutschland vor allem dem Grundsatz „Rehabilitation vor Rente“. Hinzu kommt, dass für einen international oft zu beobachtenden „Verschiebebahnhof“" von Arbeitslosigkeit in Erwerbsminderungsrente für Deutschland nur wenige Anzeichen vorliegen (so bereits Riphahn 1997; aktueller Erlinghagen und Knuth 2010 und aus qualitativer Perspektive Aurich-Beerheide et al. 2018). Allerdings gilt für Deutschland, dass sich selbst bei günstiger wirtschaftlicher Lage der Sozialleistungsbezug wegen Arbeits- bzw. Erwerbsunfähigkeit verfestigt und Übergänge in Erwerbstätigkeit nur selten gelingen (OECD 2010, S. 34).

Angesichts einer hohen Armutsquote von Erwerbsminderungsrentenbeziehenden und schlechten Aussichten, die sozioökonomische Situation aus eigener Kraft zu verbessern (Märtin und Zollmann 2011; Märtin et al. 2012, 2015), hat die Forschung Risikofaktoren für den Zugang in die Erwerbsminderungsrente untersucht und dabei auf die Qualifikation, die ausgeübte Tätigkeit, aber auch die Arbeitsmarktlage und damit insgesamt auf die unterdurchschnittliche Beschäftigungsfähigkeit, aber auch auf Beschäftigungschancen hingewiesen (Hagen et al. 2011; Müller et al. 2013; Mika 2013; Hagen und Himmelreicher 2014). Analysen der Erwerbs- und Versicherungsverläufe vor Eintritt in eine Erwerbsminderungsrente zeigen an, dass es typische Verläufe in die Erwerbsminderungsrente hinein gibt. Sie sind stark sozialrechtlich geprägt (siehe Brussig 2012; Gasche und Härtl 2013; Söhn und Mika 2015; sowie zusätzlich unter Berücksichtigung von Altersrenten Söhn und Mika 2019). So ist der Zugang in die Erwerbsminderungsrente aus dem ALG-II-Bezug zwischen 2005 und 2012 gestiegen. In dieser Zeit wurden mit dem ALG-II-Bezug versicherungsrechtliche Zeiten angesammelt, aufgrund derer es möglich war, eine Erwerbsminderungsrente zu beziehen. Erst während bzw. aufgrund des ALGII-Bezuges erfüllten ehemalige Bezieher_innen der Sozialhilfe die Anspruchsvoraussetzungen auf Erwerbsminderungsrente (Mika et al. 2014; Bäcker 2012).

Forschungsansätze, die dagegen den Grundsatz der Befristung ernst nehmen und die Erwerbsintegration nach Auslaufen einer Erwerbsminderungsrente oder weitergehende Beschäftigungsverläufe untersuchen, sind nur vereinzelt zu finden. Beispielsweise hat Briest (2018, S. 187) herausgefunden, dass sich die Motivation von Erwerbsminderungsrentner_innen, wieder in Beschäftigung zurückzukehren, zwischen den Hauptdiagnosegruppen deutlich unterscheidet. Sie ist deutlich höher bei Versicherten mit Kreislauferkrankungen $(43,8 \%)$ als bei Versicherten mit psychischen Erkrankungen (27,5\%). Die Chancen auf eine Rückkehr schätzen die Befragten ,über die Diagnosegruppen hinweg im Mittel als sehr gering [...] ein“ (ebd.). Zschucke et al. (2016, S. 227) ermittelten unter den befristeten Erwerbsminderungsrentner_innen einen Anteil von 52,0\% mit einer Rückkehrabsicht. Mit steigendem Alter und zunehmender Dauer des Rentenbezugs geht die Rückkehrabsicht jedoch zurück. Eine weitere Barriere wird darin gesehen, dass der Erwerbsminderungsrentenbezug aus einer Arbeitslosigkeit oder aus prekärer Beschäftigung heraus erfolgte, sodass eine Rückkehr in die Beschäftigung nur möglich scheint, wenn es starke Anreize für Arbeitgeber gibt. Bei den Arbeitgebern bestünde aber nur eine geringe Bereitschaft, sich mit leistungseingeschränkten Bewerber_innen auseinanderzuset- 
zen; zudem bestünden Informationsdefizite zu möglichen Rehabilitationsleistungen, was eine weitere Hürde für eine Reintegration darstellt (Zschucke und Lippke 2017).

In einer weiteren Untersuchung wurde auf der Grundlage des Scientific Use File „Abgeschlossene Rehabilitation 2006-2013 im Versicherungsverlauf“" eine Eintrittskohorte von Personen untersucht, die 2006 erstmals eine befristete Erwerbsminderungsrente erhalten haben. Von 9.919 Personen hatten sechs Jahre später noch 7.595 die Chance einer Rückkehr in den Arbeitsprozess; die übrigen waren entweder verstorben, in Altersrente übergegangen, oder ihre befristete Erwerbsminderungsrente war entfristet worden. Von diesen 7.595 Personen gelang im Zeitraum von 2007-2013 11\% eine Rückkehr in Beschäftigung. Männer erreichten häufiger eine Rückkehr in Beschäftigung als Frauen (14,5 vs. 8,4\%). Personen, denen eine Rückkehr in Beschäftigung glückte, waren im Durchschnitt sechs Jahre jünger als Personen, denen das nicht gelang (Köckerling et al. 2019, S. $191 \mathrm{f}$.).

Zusammenfassend lässt sich zum Forschungsstand festhalten, dass die Wege aus der Erwerbsminderungsrente heraus weniger untersucht wurden als die Wege in die Erwerbsminderungsrente hinein. Die vorliegenden Studien zeigen, dass zwar die Erwerbsmotivation zumindest anfänglich hoch ist, aber nur einem kleinen Teil der Erwerbsgeminderten die Rückkehr in Beschäftigung gelingt, wobei dies für Männer öfter als für Frauen und für Jüngere häufiger als für Ältere zu verzeichnen ist. Allerdings scheint der von Köckerling et al. (2019) gewählte Ansatz nicht angemessen, für einen Zeitraum von acht Jahren eine Renteneintrittskohorte zu verfolgen, da bereits die durchschnittliche Rentenlaufzeit ca. sechs Jahre beträgt. Sinnvoll wäre ein Ansatz, der nicht nur auf eine Renteneintrittskohorte abstellt, sondern vollständige Erwerbsverläufe einer Stichprobe von Erwerbstätigen bzw. Versicherten untersucht. Zudem sollten nicht nur soziodemografische Merkmale wie Alter und Geschlecht in die Untersuchung der Rückkehrchancen einbezogen werden, sondern auch erwerbsbiografische Charakteristika, um die Chancenstrukturen genauer zu untersuchen. Hierauf wird unten in den Hypothesen Bezug genommen (siehe Abschn. 4.2.2).

\section{Die Datengrundlage für die empirische Untersuchung}

\subsection{Datengrundlage: Versichertenkontenstichprobe der Rentenversicherung}

Für die folgenden empirischen Analysen wurde das Scientific Use File der Versichertenkontenstichprobe aus dem Erhebungsjahr 2015 genutzt (SUFVSKT 2015). Die Versichertenkontenstichprobe (VSKT) beruht auf prozessproduzierten Daten der Rentenversicherung (Himmelreicher und Stegmann 2008). Die Daten gelten als genau, und die Fallzahl ist mit 66.975 Fällen hoch. Über einen Hochrechnungsfaktor können sie auf die Grundgesamtheit aller Versicherten hochgerechnet werden (siehe Deutsche Rentenversicherung 2016, 2017; Stegmann 2016). Das Merkmalsspektrum ist auf Merkmale beschränkt, die für die Tätigkeit der Rentenversicherung unbedingt erforderlich sind, und ist mithin begrenzter, als für sozialwissenschaftliche Analysen wünschenswert wäre.

Die Grundgesamtheit der SUFVSKT 2015 bilden Personen, die am 31.12.2015 mindestens einen Eintrag in ihrem Versichertenkonto hatten und den Geburtsjahrgän- 
gen 1948 bis 1985 angehören. Sofern zum 31.12.2015 die Versicherten das 65. Lebensjahr noch nicht erreicht haben, sind die Daten rechtszensiert (Stegmann 2016). Für die Zwecke der vorliegenden Analyse wurde die Stichprobe eingeschränkt, indem nur Fälle mit geklärten Versichertenkonten einbezogen wurden. Zudem wurden Personen mit Versicherungszeiten nach dem Fremdrentengesetz und mit knappschaftlichen Renten ausgeschlossen. Durch diese Einschränkungen reduziert sich der Umfang des Datensatzes auf 61.532 Fälle oder hochgerechnet auf 34.935.356 Personen.

Die Stärke des Datensatzes liegt darin, dass Anfang und Ende einer Erwerbsminderungsrente beobachtet und somit die Versicherungsverläufe vor, während und nach einer Erwerbsminderungsrente untersucht werden können. Mit dem Datensatz sind aber auch Einschränkungen verbunden. Die wichtigsten Einschränkungen sind die folgenden:

- Die Versicherungsverläufe sind nur bis zum 31.12.2015 enthalten und damit rechtszensiert. Besteht zu diesem Zeitpunkt eine Erwerbsminderungsrente, kann nichts über den weiteren Versicherungsverlauf gesagt werden.

- Zum Ziehungsstichtag (31.12.2015) muss für die enthaltenen Fälle ein Versicherungskonto bestehen. Personen, die bereits verstorben sind - und mithin auch Personen, deren Erwerbsminderungsrente durch Tod beendet wurde -, sind in der Stichprobe nicht enthalten.

- Es wird nur ein Rentenbezug, aber nicht die Rentenart ausgewiesen. Erwerbsminderungsrenten lassen sich nur vor Erreichen des 60. Lebensjahres eindeutig identifizieren, da vor dem 60. Lebensjahr keine Altersrenten möglich sind. Nach dem 60. Lebensjahr sind sowohl Altersrenten als auch Erwerbsminderungsrenten möglich, sodass eine genaue Bestimmung der Rentenart nicht möglich ist. Lediglich bei Fällen, deren Rente bis zum 31.12.2015 fortbesteht, ist die Rentenart bekannt. Ebenso liegt die Information über eine Teilrente nur zum 31.12.2015 vor. Diese Fälle sind aber rechtszensiert und lassen sich nicht auswerten.

- Es gibt für die Zeiten der Beschäftigung nur Informationen zum Entgelt, aber nicht zur Wochenarbeitszeit. Es lässt sich also nicht unterscheiden, ob Änderungen des Monatsentgelts mit Änderungen des Stundenlohns oder der Wochenarbeitszeit zusammenhängen.

Trotz dieser Einschränkung kann die VSKT aufgrund ihrer Größe, Genauigkeit und der vorhandenen Merkmale grundsätzlich als geeigneter Datensatz angesehen werden. 


\subsection{Hypothesen und Variablen}

\subsubsection{Die abhängige Variable: Identifikation von Phasen mit Erwerbsminderungsrentenbezug und des Zustands nach einer Erwerbsminderungsrente}

Wie oben dargelegt, wird die Rentenart (Rente wegen Alters oder wegen verminderter Erwerbsfähigkeit) in den relevanten Daten ${ }^{5}$ nicht eindeutig ausgewiesen. Da vor dem 60. Lebensjahr Altersrenten nicht möglich sind, werden Phasen des Rentenbezugs vor dem 60. Lebensjahr aus eigener Versicherung als Erwerbsminderungsrenten gewertet. Mit Vollendung des 60. Lebensjahres wird in der hier vorliegenden Analyse ein Ende der Erwerbsminderungsrente aus Altersgründen angenommen. Hierfür sprechen sowohl ein faktischer als auch ein datentechnischer Grund: Erstens kommt es jenseits des 60. Lebensjahres nur sehr selten nach einer Erwerbsminderungsrente zu einem Wechsel in Beschäftigung (oder überhaupt zu einem Ende des Rentenbezuges), und zweitens lässt sich (wegen der fehlenden Angabe zur Leistungsart) in der Regel nicht eindeutig bestimmen, wann eine Umwandlung der Erwerbsminderungsrente in eine Altersrente erfolgte.

Es ist möglich, dass eine Person parallel zum Erwerbsminderungsrentenbezug einer Erwerbstätigkeit nachgeht. Dies kann insbesondere bei einer Rente wegen teilweise geminderter Erwerbsfähigkeit auftreten. Personen, die parallel zum Bezug einer Erwerbsminderungsrente beschäftigt sind, werden hier als Erwerbsminderungsrentner_innen gewertet. Dies ist vor allem bedeutsam für die Untersuchung von Übergängen von einer Erwerbsminderungsrente in Beschäftigung. Wenn eine Person während eines Bezugs einer Erwerbsminderungsrente eine Beschäftigung aufnimmt, dann ist das noch kein Weg aus der Erwerbsminderungsrente heraus und wird deshalb hier nicht als Beschäftigung nach einer Erwerbsminderungsrente gewertet. Ein Übergang in Beschäftigung im Sinne der vorliegenden Untersuchung liegt erst dann vor, wenn der Erwerbsminderungsrentenbezug beendet und die Beschäftigung fortgeführt wird.

Abb. 3 stellt diese beiden typischen Verläufe dar und veranschaulicht die Implikationen der hier verwendeten Definitionen. Es sind jeweils zwei Stränge dargestellt, von denen ein Strang den Rentenbezug markiert und ein anderer Strang die Erwerbstätigkeit. Im Fall 1 endet eine Erwerbsminderungsrente, und nach einer gewissen Zeit, die in dem Beispiel länger als sechs und kürzer als zwölf Monate dauert, wird eine Beschäftigung aufgenommen, die später wieder endet. In Fall 2 läuft parallel zum Bezug der Erwerbsminderungsrente eine Beschäftigung. Auch dieser Fall wird als ein Übergang aus der Erwerbsminderungsrente in Beschäftigung gewertet, allerdings erst ab dem Zeitpunkt, nachdem der Bezug einer Erwerbsminderungsrente endete.

Für die 61.532 Fälle, die in die Untersuchung einbezogen werden, lassen sich 24.894.840 monatliche Meldungen aus ihren Versicherungsbiografien auswerten.

\footnotetext{
5 Die Leistungsart ist nur zum Stichtag (31.12.2015) ausgewiesen und betrifft deshalb zensierte Rentenbezugsinformationen, für die keine Information zum Ende des Rentenbezugs und zum Versicherungsstatus danach vorliegt. Zensierte Fälle gehen daher nicht in die Auswertung ein.
} 


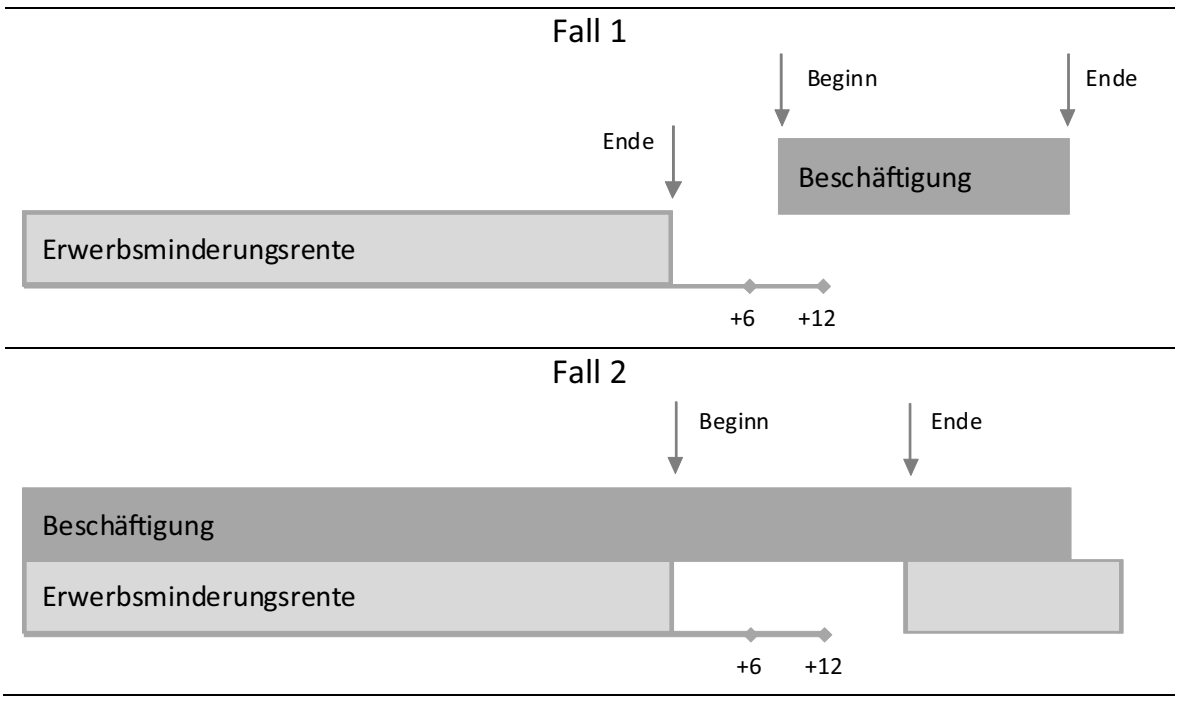

Abb. 3 Typische Verläufe von Rentenbezug und Beschäftigung. (Quelle: Eigene Darstellung)

Von diesen beinhalten 312.623 Meldungen Monate mit einer Erwerbsminderungsrente. Mehrere aufeinander folgende Monate mit Erwerbsminderungsrentenbezug werden zu einer Rentenepisode zusammengefasst. ${ }^{6}$ Die Auswertung umfasst 3.501 Episoden von 3.186 Personen. Hochgerechnet durchleben von den knapp 35 Mio. im Datensatz enthaltenen Versicherten knapp 1,9 Mio. mindestens eine Phase mit einem Erwerbsminderungsrentenbezug. Die Gesamtzahl der Rentenepisoden aufgrund einer Erwerbsminderung liegt hochgerechnet bei gut 2,1 Mio.

Knapp $72 \%$ der Rentenepisoden aufgrund einer Erwerbsminderung sind zensiert, d.h. sie enden am 31.12.2015.7 Diese Fälle können nicht in die Untersuchung einbezogen werden. Die Auswertungen beziehen sich auf abgeschlossene Erwerbsminderungsrenten. Dies betrifft etwa 28\% der Episoden mit Bezug einer Erwerbsminderungsrente (also 869 Episoden). Diese 869 Episoden stammen von 736 unterschiedlichen Personen.

\subsubsection{Hypothesen und unabhängige Variablen: Personenmerkmale und Merkmale der Erwerbsbiografie}

An Personenmerkmalen aus dem fixen Datenteil werden das Geburtsjahr, das Geschlecht und die berufliche Qualifikation verwendet, weil zu erwarten ist, dass die Rückkehrhäufigkeit in Beschäftigung nach einer Erwerbsminderung mit dem Alter, Geburtsjahr, Geschlecht und der Qualifikation korreliert. Beschäftigung, Arbeits-

\footnotetext{
6 Rentenepisoden, die durch maximal drei Monate mit anderen Erwerbszuständen unterbrochen waren, wurden zusammengefasst.

7 Zudem liegt bei gut zwei Prozent der Fälle keine Information über den Folgezustand vor.
} 
losigkeit und Entgeltpunkte ${ }^{8}$ liegen als Merkmale der Erwerbsbiografie als Verlaufsvariable vor. Es gehört zu den Stärken der VSKT, dass diese Daten in ihrem monatsgenauen Verlauf vorliegen.

Aufgrund einer typischerweise höheren Erwerbsorientierung von Männern gegenüber Frauen (Franz et al. 2012; Kümmerling et al. 2015; Bauer et al. 2016; Lott 2017) bzw. der Möglichkeit insbesondere für Frauen, ,Alternativrollen“ (Offe und Hinrichs 1977) wie Sorgearbeit oder Hausfrauenarbeit zu nutzen, ist zu erwarten, dass die Beschäftigungschance der Männer nach einer Erwerbsminderungsrente höher ist als die von Frauen (Hypothese 1). Erwartbar ist, dass bei jüngeren Erwerbsgeminderten aufgrund der längeren Lebensphase bis zur Altersrente die Erwerbsmotivation c.p. höher ist als bei älteren. Möglich ist auch, dass bei älteren Erwerbsgeminderten weitere Erkrankungen bestehen - über die Erkrankung bzw. Behinderung hinaus, die der Erwerbsminderung zugrunde liegt -, welche die Beschäftigungschancen verringern, oder dass ältere Personen mit gesundheitlichen Einschränkungen bereitwilliger verrentet werden. Beide Überlegungen sprechen dafür, dass Jüngere häufiger nach einer befristeten Erwerbsminderungsrente in Beschäftigung kommen (Köckerling et al. 2019; Lohse und Rodriguez Gonzalez 2015; Schneider 2007; Eriksson und Lagerström 2012) (Hypothese 2).

Das Geburtsjahr ist ein Indikator dafür, nach welchem Recht eine Erwerbsminderungsrente zugesprochen wird. Wie oben dargestellt, besteht für Personen, die vor dem 02.01.1961 geboren wurden, ein Berufsschutz. Diese Regelung betrifft den Eintritt in die Rente und nicht die Chancen nach deren Beendigung. Aufgrund der restriktiveren Bewilligung nach der Reform (also für Personen, die ab dem 02.01.1961 geboren wurden) ist zu vermuten, dass auch eine Verlängerung der Erwerbsminderungsrente restriktiver gehandhabt wird, zumal erst mit der Reform die Befristung als Regelfall gehandhabt wurde (siehe oben). In der Folge dürften die Erwerbschancen der „reformbetroffenen“ Personen schlechter sein als die von Personen, über deren Verlängerung großzügiger entschieden wird, weil Letztere bei schlechten Beschäftigungschancen tendenziell öfter in Rente verbleiben dürften, während die ,,reformbetroffenen" Personen trotz schlechter Beschäftigungschancen tendenziell öfter befristet werden (Hypothese 3).

Für die Qualifikation lassen sich Gründe finden, die für bzw. gegen eine häufigere Erwerbsintegration von höher Qualifizierten gegenüber geringer Qualifizierten sprechen. Höher qualifizierte Personen haben einerseits grundsätzlich mehr Beschäftigungsmöglichkeiten, weil sie nicht auf Tätigkeiten auf ihrem Qualifikationsniveau beschränkt sind, sondern darüber hinaus weitere un- und angelernte Tätigkeiten verrichten können (Hypothese $4 \mathrm{a}$ ). $\mathrm{Zu}$ vermuten ist, dass bei höher qualifizierten Erwerbsminderungsrentner_innen die Spielräume für Arbeitsplatzanpassungen höher sind als bei Geringqualifizierten, was deren Rückkehrchancen bei bestehenden Beschäftigungsverhältnissen vergrößert. Zudem signalisieren höhere Qualifikatio-

\footnotetext{
8 Die Entgeltpunkte markieren den in einem Zeitraum erworbenen Rentenanspruch, der sich anhand von Beitragszahlungen bemisst. Die Entgeltpunkte enthalten also eine Information zum Entgelt und können bis zur Beitragsbemessungsgrenze für einen Vergleich der versicherten Einkommen genutzt werden. Entgelte oberhalb der Beitragsbemessungsgrenze werden nicht für Rentenbeiträge herangezogen und bleiben daher für die Entgeltpunkte unberücksichtigt.
} 
nen eine höhere Produktivität und Leistungsbereitschaft, die sie für die Arbeitgeber attraktiver machen. Insgesamt verfügen höher Qualifizierte über bessere Arbeitsmarktchancen als Geringqualifizierte, was sich deutlich in qualifikationsspezifischen Arbeitslosenquoten zeigt, die für höher Qualifizierte niedriger sind als für Geringqualifizierte (Erlinghagen 2006, 2017; Solga 2014; Autorengruppe Bildungsberichterstattung 2018).

Gegen bessere Erwerbschancen der höher Qualifizierten gegenüber Geringqualifizierten spricht, dass sich der Rentenanspruch in der Erwerbsminderungsrente aus einer Fortschreibung des bisherigen Entgelts bestimmt (,Zurechnungszeiten“" ${ }^{\prime \prime}$ ). Höher Qualifizierte können daher eine höhere Rente mit Fortführung der Erwerbsminderungsrente erhalten als sie durch eine unterwertige Beschäftigung erzielen, und dies würde langfristig den künftigen Altersrentenanspruch schmälern. Und umgekehrt könnte für geringer Qualifizierte ein niedriger Rentenanspruch als Treiber für eine Beschäftigungsaufnahme wirken. Vor diesem Hintergrund ist es auch möglich, dass geringer Qualifizierte häufiger nach einer Erwerbsminderungsrente eine Beschäftigung aufnehmen als höher Qualifizierte (Hypothese 4b).

Gerade die Privilegierung der höher Qualifizierten gegenüber den Geringqualifizierten vor der Reform durch die Berufsunfähigkeitsrente war seit langem eines der stärksten Argumente für ihre Abschaffung 2001 (Ruland und Rische 1980; Ruland 1990). Aus den gleichen Gründen lässt sich auch für die Einkommensposition vor der Erwerbsminderungsrente sowohl eine höhere (Hypothese 5a) als auch eine niedrigere Beschäftigungswahrscheinlichkeit nach einer Erwerbsminderungsrente vermuten (Hypothese 5b).

Personen mit einer stabilen Erwerbsbiografie tragen in geringerem Ausmaß Arbeitsmarktrisiken (Erlinghagen 2008, 2017; Krause und Köhler 2012; Kirchner 2016), und es ist deshalb zu vermuten, dass dies auch für die Folgen einer Erwerbsminderung gilt, d.h. dass die Aufnahme einer Beschäftigung nach einer Erwerbsminderungsrente häufiger bei Personen zu erwarten ist, die vor der Erwerbsminderungsrente stabil beschäftigt waren (Hypothese 6).

Hinsichtlich der Dauer der Erwerbsminderungsrente ist zu vermuten, dass mit zunehmender Dauer die Wahrscheinlichkeit einer Beschäftigungsaufnahme zurückgeht, weil Arbeitserfahrungen und Arbeitsmotivation verloren gehen (Zschucke et al. 2016; Zschucke und Lippke 2017; Behrens 1994; Schubert et al. 2006) (Hypothese 7); ähnliche Entwertungen von Kompetenzen und Motivation sind bei Arbeitslosigkeit vielfach belegt (Dieckhoff 2011; Gabriel et al. 2013; Lietzmann et al. 2018; Bauer und Sieglen 2018; Paul und Moser 2019).

Außerdem lässt sich bestimmen, ob parallel zum Bezug einer Erwerbsminderungsrente eine Beschäftigung vorlag. Zu vermuten ist, dass einige Beschäftigungsmonate während des Erwerbsminderungsrentenbezugs - insbesondere an deren En-

\footnotetext{
${ }^{9}$ Die Rentenhöhe wird bei Erwerbsminderungsrenten aus den bislang erworbenen Rentenansprüchen sowie sogenannten Zurechnungszeiten ermittelt. Mittels der Zurechnungszeiten wird unterstellt, dass sich die bis zum Zeitpunkt der Erwerbsminderung realisierte Versicherungsbiografie bis zum Erreichen einer Altersgrenze fortgesetzt hätte, ab der auch eine Altersrente bezogen werden kann. Insofern ist in der Erwerbsminderungsrente das für das deutsche Rentensystem charakteristische Äquivalenzprinzip enthalten, aber auch ein starkes Element des sozialen Ausgleichs, da die Beiträge nur für einen Teil der Erwerbsbiografie und nicht bis zur Regelaltersgrenze entrichtet wurden.
} 
Tab. 1 Übersicht über die Hypothesen. (Quelle: Eigene Darstellung)

\begin{tabular}{|c|c|}
\hline & Höhere Beschäftigungschance nach einer Erwerbsminderungsrente ... \\
\hline $\mathrm{H} 1$ & bei Männern \\
\hline $\mathrm{H} 2$ & mit niedrigerem Alter \\
\hline H3 & $\begin{array}{l}\text { wenn nicht von der Reform der Erwerbsminderungsrente } 2001 \text { betroffen (d. h. älter als Jahr- } \\
\text { gang 1961) }\end{array}$ \\
\hline $\mathrm{H} 4$ & bei hoher oder niedriger Qualifikation (beide Richtungen denkbar) \\
\hline H5 & $\begin{array}{l}\text { bei hoher oder niedriger Einkommensposition vor der Erwerbsminderungsrente (beide Rich- } \\
\text { tungen denkbar) }\end{array}$ \\
\hline H6 & wenn stabil beschäftigt vor der Erwerbsminderungsrente \\
\hline $\mathrm{H} 7$ & bei kürzerer Dauer der Erwerbsminderungsrente \\
\hline H8 & wenn bereits vor Ende der Erwerbsminderungsrente beschäftigt \\
\hline
\end{tabular}

de - positiv mit den Übergangschancen in Beschäftigung nach der Erwerbsminderungsrente korrelieren (Hypothese 8), da die Leistungsfähigkeit der betreffenden Person im Betrieb hinlänglich bekannt ist und damit ein verbreiteter Vorbehalt gegen die Beschäftigung von Personen mit gesundheitlichen Einschränkungen ausfällt (Wuppinger und Rauch 2010; BA 2017). Die Hypothesen sind zusammenfassend in Tab. 1 dargestellt.

\section{Wege aus der Erwerbsminderungsrente}

\subsection{Versicherungsstatus nach dem Ende einer Erwerbsminderungsrente}

Die zentrale Frage der vorliegenden Analyse ist, wovon es - im Rahmen des beobachtbaren Merkmalsspektrums - abhängt, dass Versicherte nach Ende einer Erwerbsminderungsrente in Beschäftigung sind. Hierfür ist zu klären, welcher Zeitpunkt herangezogen werden soll, zu dem der Erwerbsstatus beobachtet wird. Dieser Zeitpunkt sollte nicht zu dicht nach dem Ende des Erwerbsminderungsrentenbezugs liegen, sondern eine gewisse „Suchzeit“" vorsehen. Er sollte aber auch nicht zu weit entfernt sein, da nach dem Ende des Rentenbezugs die Erwerbsfähigkeit wiederhergestellt und in einem überschaubaren Zeitraum verwirklicht sein sollte.

Der häufigste Grund, aus dem eine Erwerbsminderungsrente in unserem Untersuchungsfall endet, ist die Vollendung des 60. Lebensjahres (48,3\%, siehe Abb. 4). Es ist zu betonen, dass dieses Ende bzw. dieser Grund pragmatisch für die Zwecke der vorliegenden Untersuchung gesetzt wurde. Wie oben dargelegt, ist jenseits dieses Alters nicht mehr zu entscheiden, welche Rentenart bezogen wird und wann eine Umwandlung in Altersrente erfolgt. Nur in sehr wenigen Fällen wird ein Rentenbezug jenseits des 60. Lebensjahres noch unterbrochen.

In dem Zwölf-Monats-Zeitraum, der auf das Ende der Erwerbsminderungsrente folgt, steigt der Anteil jener, die in eine Altersrente wechseln. Diese Personen kommen vollständig aus dem Kreis jener, für die zuvor ein Ende der Erwerbsminderungsrente altersbedingt (mit 60 Jahren) festgestellt wurde. Es steigt aber auch der Anteil jener, die nach dem Ende einer Erwerbsminderungsrente (vor Vollen- 


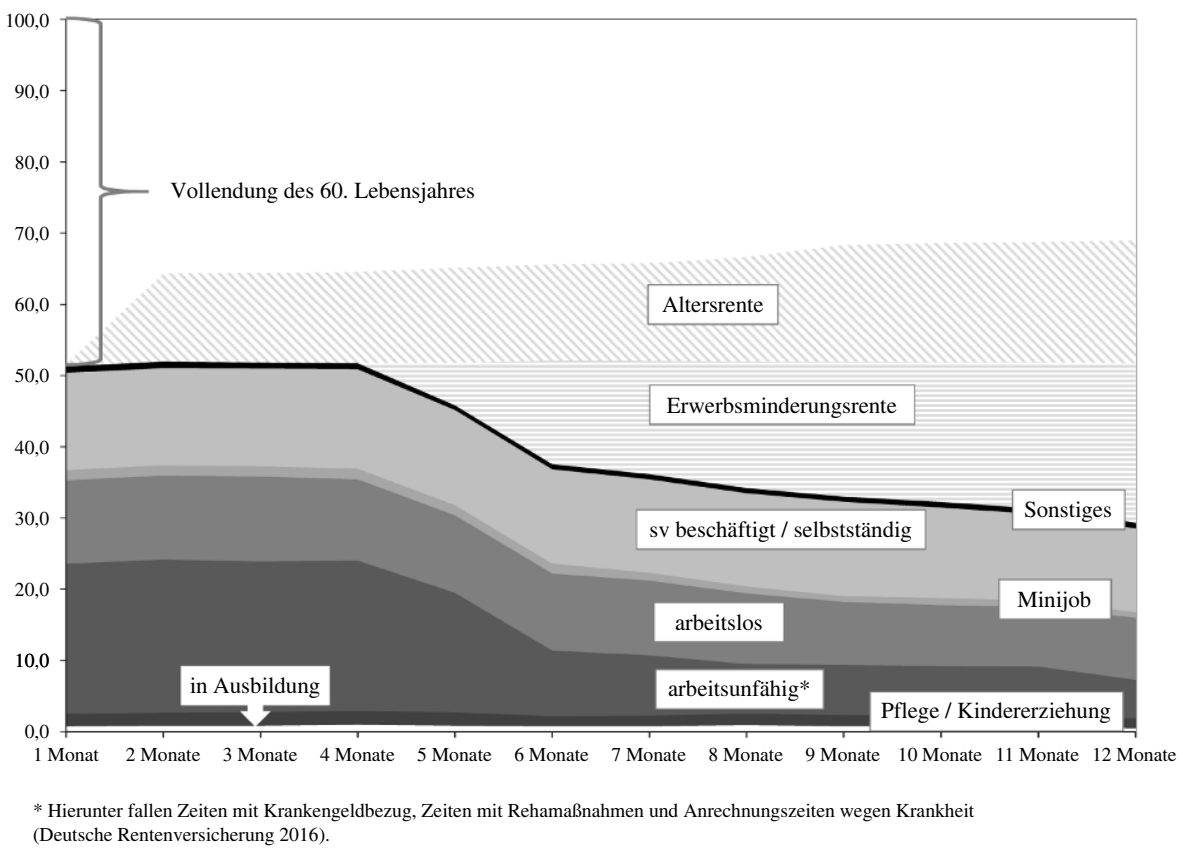

Abb. 4 Versicherungsstatus in den zwölf Monaten nach Ende einer Erwerbsminderungsrente (Anteil an allen Übergängen in Prozent). (Quelle: SUFVSKT 2015; eigene Berechnungen, gewichtet)

dung des 60. Lebensjahres) erneut eine Erwerbsminderungsrente beziehen; dies trifft nach zwölf Monaten auf 22,4\% zu. Von den Personen, deren Bezug einer Erwerbsminderungsrente endete, sind über zwei Drittel (70,7\%) ein Jahr später entweder mindestens 60 Jahre alt (und beziehen eine Rente) oder beziehen (erneut) eine Erwerbsminderungsrente.

Im Monat nach Ende des Bezugs einer Erwerbsminderungsrente sind 15,0\% der Versicherten in einem Minijob, einer versicherungspflichtigen Beschäftigung oder einer rentenversicherten selbstständigen Tätigkeit. Hier kann es sich sowohl um die Fortführung einer bereits ausgeübten Tätigkeit als auch um eine Beschäftigungsaufnahme handeln. Zwölf Monate nach Ende der Erwerbsminderungsrente ist der Anteil an Erwerbstätigen mit rund $12,5 \%$ etwas niedriger. Rechnet man noch jene Personen hinzu, die arbeitslos gemeldet sind (8,7\%), ist zu diesem Zeitpunkt jede_r Fünfte der ehemaligen Erwerbsminderungsrentner_innen erwerbsbeteiligt oder strebt eine Beschäftigung unmittelbar an.

Vergleicht man den Erwerbs- bzw. Versicherungszustand sechs und zwölf Monate nach einer Erwerbsminderungsrente, so zeigt sich, dass die meisten Personen nach zwölf Monaten im selben Erwerbs- bzw. Versicherungszustand wie nach sechs Monaten sind (siehe Tab. 2).

In einigen Erwerbszuständen ist jedoch eine höhere Fluktuation zu verzeichnen. Von den Versicherten, die sechs Monate nach Ende einer Erwerbsminderungsrente in einem Minijob sind, verbleiben nur gut zwei Drittel in einem Minijob (68,5\%). Die Übrigen wechseln zurück in eine Erwerbsminderungsrente oder aber in eine 


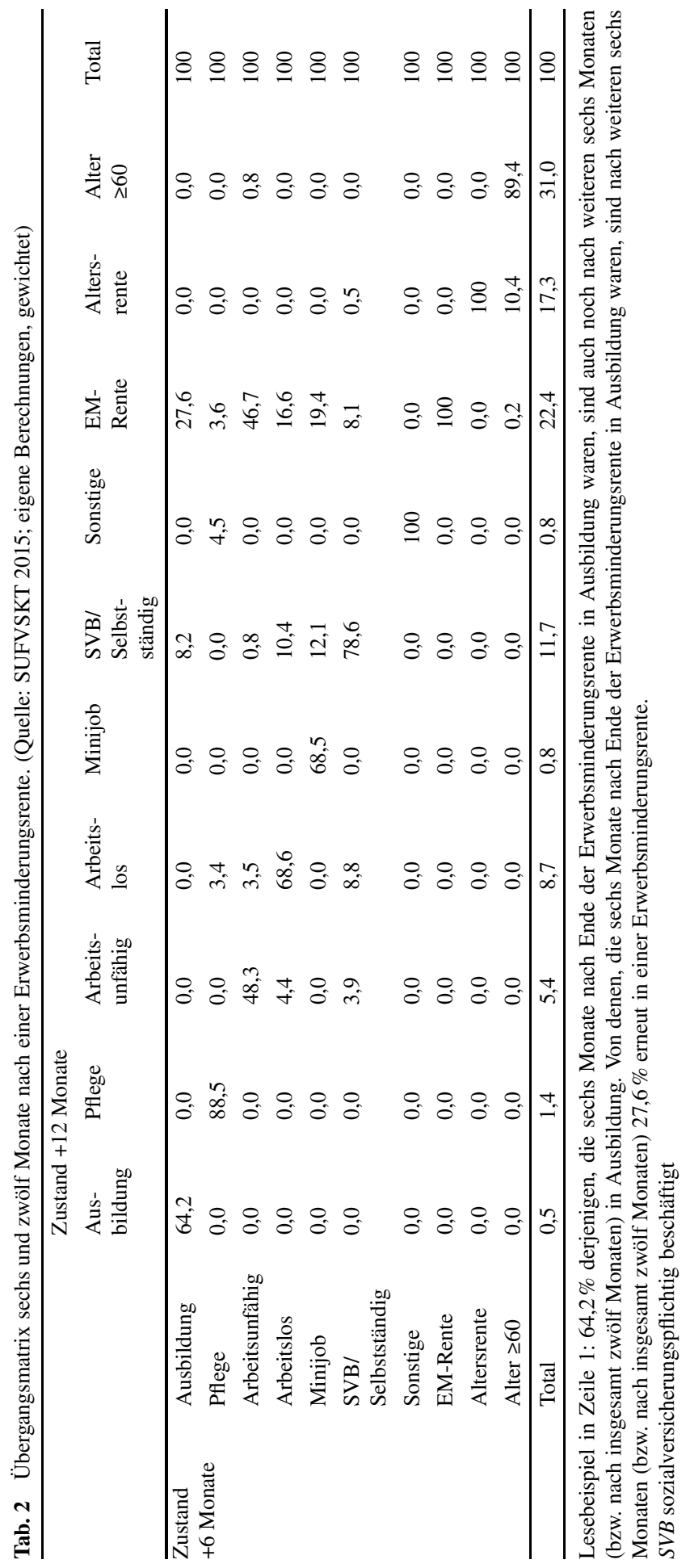


versicherungspflichtige Beschäftigung. Ebenso hoch ist die Fluktuation bei jenen, die nach sechs Monaten arbeitslos sind. Von ihnen verbleiben zwei Drittel (68,6\%) in diesem Zustand. Fast jede_r Sechste $(16,6 \%)$ wechselt dagegen zurück in eine Erwerbsminderungsrente. Immerhin gut zehn Prozent nehmen eine versicherungspflichtige Beschäftigung auf.

Versicherungspflichtig Beschäftigte und versicherte Selbstständige sind demgegenüber in ihrem Status stabiler als arbeitslose oder geringfügig beschäftigte Personen. Sie verbleiben zwischen dem sechsten und zwölften Monat nach dem Ende des Erwerbsminderungsrentenbezugs zu fast vier Fünfteln in versicherungspflichtiger Beschäftigung (78,6\%). Ein großer Teil von ihnen war bereits direkt nach dem Ende der Erwerbsminderungsrente - und vermutlich bereits während des Rentenbezuges - erwerbstätig.

Am höchsten ist die Fluktuation bei Personen, die sechs Monate nach einer Erwerbsminderungsrente arbeitsunfähig sind. Davon ist nur noch knapp die Hälfte nach zwölf Monaten in diesem Zustand (48,3\%). Nahezu ebenso viele (46,7\%) beziehen wieder eine Erwerbsminderungsrente. Knapp vier Prozent sind arbeitslos und knapp ein Prozent hat eine Beschäftigung aufgenommen. Somit kann man die Arbeitsunfähigkeit nach einer Erwerbsminderungsrente - gerade im Vergleich zu Arbeitslosigkeit - trotz der sozialrechtlich bescheinigten Erwerbsfähigkeit nicht als einen arbeitsmarktnahen Zustand sehen. Es scheint eher ein beträchtliches Segment der Versicherten mit Latenz- oder Unsicherheitsphasen zu geben, die zwischen Erwerbsminderungsrente und Arbeitsunfähigkeit wechseln.

Für die Ermittlung von Übergangswahrscheinlichkeiten in Beschäftigung ist es wichtig, einen Zeitpunkt zu bestimmen, zu dem ein Erwerbszustand aussagekräftig für den weiteren Erwerbsverlauf ist. Befindet sich jemand zwölf Monate nach einer Erwerbsminderungsrente in Beschäftigung, ist diese stabiler als ein Beschäftigungsverhältnis nach sechs Monaten. Von den Beschäftigungsverhältnissen, die nach sechs Monaten bestehen, haben knapp 76\% eine Dauer von zwölf und mehr Monaten. Bei den Beschäftigungsverhältnissen nach zwölf Monaten trifft dies auf knapp $98 \% \mathrm{zu}$. Mit wachsendem zeitlichen Abstand zum Ende der Erwerbsminderungsrente werden die Beschäftigungsverhältnisse also stabiler. Dies deutet auf einen Sortierprozess hin, in dem die Beschäftigten Arbeitsplätze suchen bzw. Arbeitgeber Arbeitsplätze bereitstellen, die von den Beschäftigungssuchenden mit den gegebenen Leistungsvermögen besetzt werden können. Hier scheint es häufig zu Fehleinschätzungen zu kommen, sodass Beschäftigungsverhältnisse aufgelöst und anderswo neu begonnen werden. Ob diese neuen Beschäftigungsverhältnisse im selben Betrieb und im selben Beruf liegen, lässt sich den Daten nicht entnehmen, und ebenso wenig, ob die Beschäftigungsverhältnisse durch die Versicherten oder durch die Arbeitgeber aufgelöst wurden. Deutlich wird aber, dass dieser Sortierprozess nicht nur in eine andere Beschäftigung hineinführt oder in eine erneute Erwerbsminderungsrente mündet, sondern ebenso häufig zu Arbeitslosigkeit führt. 


\subsection{Differenzierung der Beschäftigungschancen nach Alter, Geschlecht und Qualifikation}

In diesem Abschnitt werden die verfügbaren Personenmerkmale in ihrer Verteilung und in ihrem bivariaten Zusammenhang mit dem Erwerbsstatus nach Ende einer Erwerbsminderungsrente ausgewertet.

Die Auswertung nach Geschlecht zeigt zwischen Männern und Frauen bei den Übergangswahrscheinlichkeiten in Beschäftigung mit 12,3\% bzw. 12,8\% keinen relevanten Unterschied (vgl. Abb. 5). Frauen machen 52,5\% der Untersuchungspopulation aus.

Das Durchschnittsalter beim Beginn einer Erwerbsminderungsrente beträgt ca. 50 Jahre. Die Mehrheit der Versicherten ist am Ende ihres Erwerbsminderungsrentenbezugs mindestens 56 Jahre alt (knapp 54\%). Nur etwa jede_r Fünfte ist höchstens 40 Jahre alt (knapp 19\%). Bei Versicherten im Alter von 40 Jahren und jünger ist die Wahrscheinlichkeit, ein Jahr nach der Erwerbsminderungsrente in Beschäftigung zu sein, mit gut $32 \%$ weit überdurchschnittlich. In der mittleren Altersgruppe von 41 bis 55 Jahren ist die Beschäftigungschance mit gut $18 \%$ immer noch überdurchschnittlich, hingegen bei Älteren ab 56 Jahren mit knapp drei Prozent weit unterdurchschnittlich.

Die seltenere Aufnahme einer Beschäftigung bei Älteren korrespondiert mit häufigen Übergängen in eine Rente (siehe Tab. 3). Übergänge in Beschäftigung sind bei Jüngeren gleichbedeutend mit Übergängen in versicherungspflichtige Beschäftigung. Bis zum Alter von 55 Jahren spielen Übergänge in Minijobs kaum eine Rolle. Bei den Ältesten sind Übergänge in Minijobs und in eine versicherungspflichtige Beschäftigung gleich häufig, allerdings beide auf einem sehr niedrigen Niveau. Ebenso

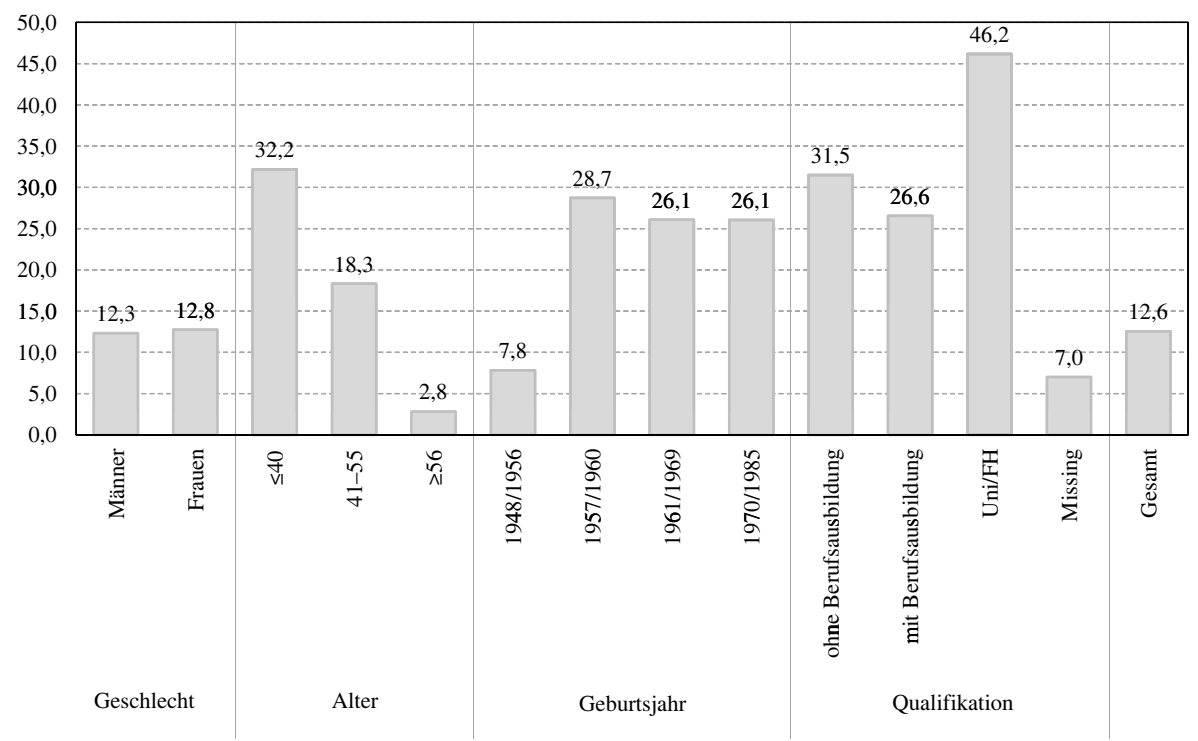

Abb. 5 Beschäftigungswahrscheinlichkeit zwölf Monate nach Ende einer Erwerbsminderungsrente nach Personenmerkmalen (in Prozent). (Quelle: SUFVSKT 2015; eigene Berechnungen, gewichtet) 


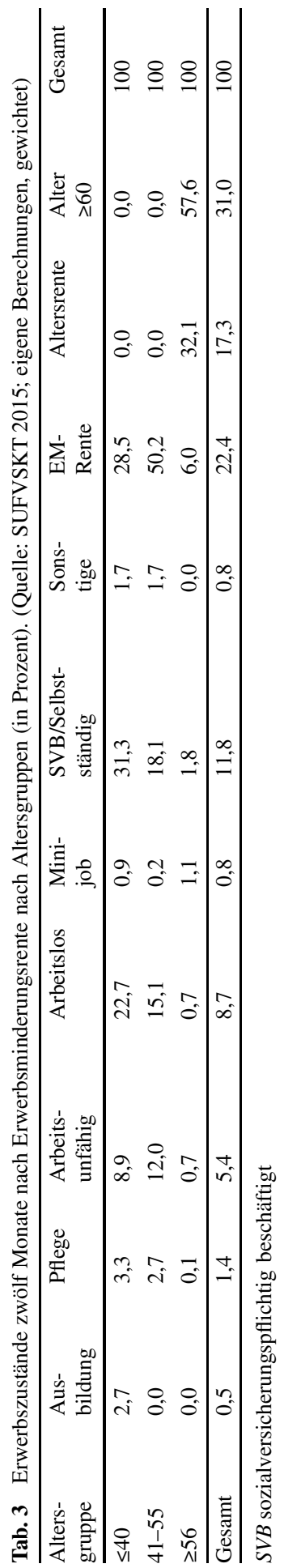


wie die versicherungspflichtige Beschäftigung ist bei Jüngeren zwölf Monate nach der Erwerbsminderungsrente auch die Arbeitslosigkeit mit knapp 23\% viel häufiger als bei Älteren mit unter einem Prozent.

Wie in Abschn. 2.2 erläutert, sind die Regelungen zur Erwerbsminderungsrente seit der Reform 2001 stärker als zuvor vom Aktivierungsparadigma geprägt. Für Versicherte, die nach dem 01.01.1961 geboren sind, bestehen stärkere Anreize zur Aufnahme einer Beschäftigung. Möglich ist also ein Kohorteneffekt in der Übergangswahrscheinlichkeit. Vergleicht man die Beschäftigungschancen der Jahrgänge von 1957 bis 1960 und ab 1961 bis 1969, d.h. vor und nach der Reform, waren die Chancen, eine neue Beschäftigung zu finden, für die früher Geborenen mit knapp $29 \%$ etwas höher als für die später Geborenen mit gut $26 \%$ (siehe Abb. 5). Mit rund $75 \%$ stammt die überwiegende Mehrheit der Untersuchungsfälle aus den Geburtsjahrgängen zwischen 1948 und 1956, für die die Übergangswahrscheinlichkeit in Beschäftigung besonders niedrig ist. Bedenkt man, dass diese Jahrgänge mittlerweile schon überwiegend das übliche Zugangsalter für Altersrenten erreicht haben, sollte bei der multivariaten Analyse (vgl. Abschn. 5.4) ein besonderes Augenmerk darauf gelegt werden, ob neben dem Alterseffekt ein eigenständiger Kohorteneffekt feststellbar ist.

Die Chance, nach einer Erwerbsminderungsrente wieder in Beschäftigung zu kommen, ist für Versicherte mit einer abgeschlossenen Berufsausbildung mit knapp $27 \%$ am niedrigsten. Für Versicherte ohne Berufsausbildung ist sie mit 31,5\% etwa fünf Prozentpunkte höher. Bei Universitäts- oder Fachhochschul-Absolvent_innen ist die Chance, wieder in Beschäftigung zu kommen, mit gut $46 \%$ am höchsten. Allerdings ist die Auswertung nach Qualifikation problematisch, weil in über 74\% der Fälle Angaben zur Qualifikation fehlen. Fälle ohne Angabe zur Qualifikation liegen mit einer Übergangswahrscheinlichkeit von sieben Prozent weit unter dem Gesamtwert von 12,6\%, während alle Fälle mit einer Angabe zum Qualifikationsniveau deutlich über dem Gesamtwert liegen. Die Auswertung nach Qualifikation kann daher zwar grobe Anhaltspunkte zu Unterschieden in den Eingliederungswahrscheinlichkeiten nach Qualifikation geben, aber die absoluten Wahrscheinlichkeiten werden überschätzt.

\subsection{Differenzierung von Beschäftigungschancen nach erwerbsbiografischen Merkmalen}

Dauert der Bezug einer Erwerbsminderungsrente nur bis zu einem Jahr, erfolgt mit gut neun Prozent nur selten ein Übergang in Beschäftigung (siehe Abb. 6). Versicherte mit sehr kurzen Erwerbsminderungsrentenphasen von höchstens einem Jahr sind zwölf Monate nach Ende des Rentenbezuges zu gut $57 \%$ wieder in einer neuen Phase des Bezugs einer Erwerbsminderungsrente und zu gut $12 \%$ in Arbeitsunfähigkeit oder Krankheit.

Dass auf kurze Erwerbsminderungsrentenphasen oft erneute Rentenphasen folgen, deutet auf eine Zeit der Unsicherheit oder Latenz hin, in der Versicherte zwischen Erwerbsminderung und Arbeitsunfähigkeit wechseln. Dies ist bereits in Abschn. 5.1 deutlich geworden, als die Auswertungen zum Erwerbsstatus nach dem Ende einer Erwerbsminderungsrente die Instabilität von Beschäftigung und Arbeits- 


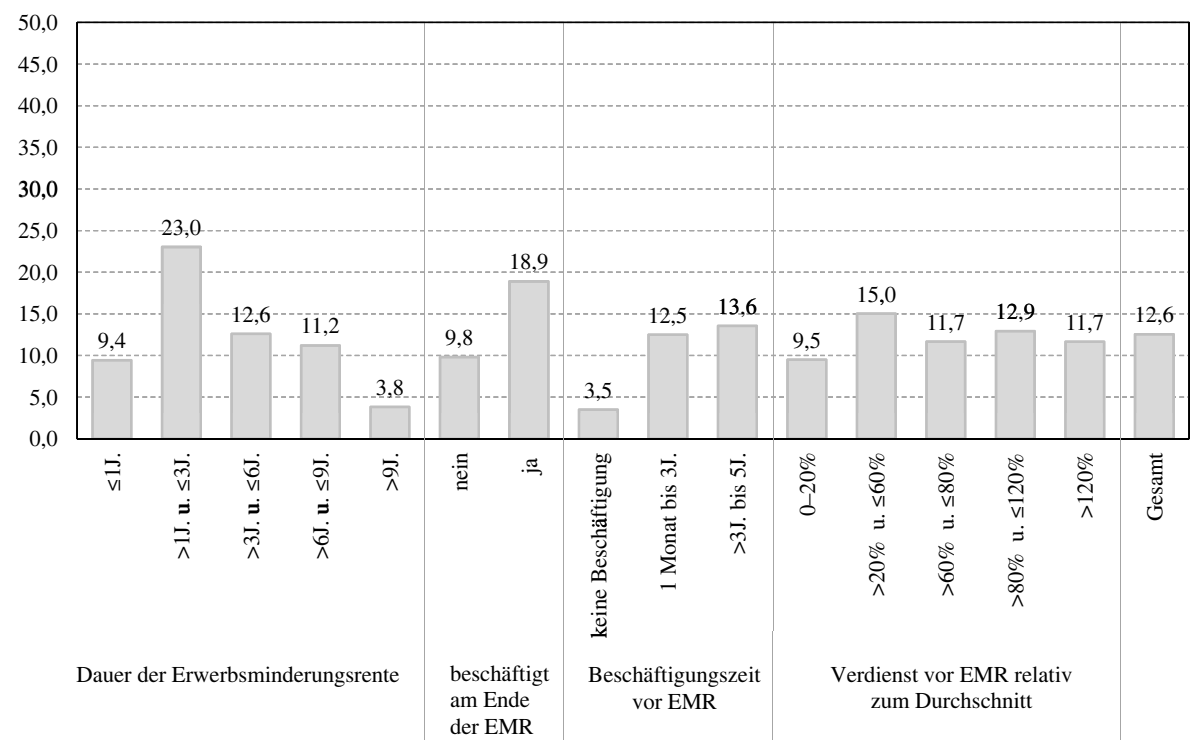

Abb. 6 Übergangswahrscheinlichkeit in Beschäftigung zwölf Monate nach Ende der Erwerbsminderungsrente nach erwerbsbiografischen Merkmalen (in Prozent). (Quelle: SUFVSKT 2015; eigene Berechnungen, gewichtet)

unfähigkeit nach einem bzw. sechs Monaten gezeigt haben. Hingegen ist nach Erwerbsminderungsrenten mit einer Dauer von über einem Jahr und bis zu drei Jahren die Beschäftigungswahrscheinlichkeit mit $23 \%$ am höchsten. Ab einer Dauer von mehr als drei Jahren geht die Beschäftigungschance immer weiter zurück bis auf knapp vier Prozent bei einer Dauer von mehr als neun Jahren. Der geringen Wiedereingliederungschance nach sehr kurzen Erwerbsminderungsrentenphasen kommt insofern eine große Bedeutung zu, als mit $35 \%$ mehr als jede_r dritte Versicherte davon betroffen ist.

Gut $63 \%$ der Bezieher_innen von Erwerbsminderungsrenten waren in den fünf Jahren vor der Erwerbsminderungsrente mehr als drei Jahre beschäftigt. Lediglich knapp sieben Prozent waren keinen einzigen Monat in Beschäftigung. Von denjenigen, die in den fünf Jahren vor einer Erwerbsminderungsrente mehr als drei Jahre in Beschäftigung waren, sind zwölf Monate nach Ende der Erwerbsminderungsrente fast $14 \%$ wieder in Beschäftigung. Von jenen, die in den fünf Jahren vor der Erwerbsminderungsrente gar nicht beschäftigt waren, ist zwölf Monate nach dem Ende der Erwerbsminderungsrente nur ein Anteil von 3,5\% wieder in Beschäftigung. Deskriptiv bestätigt sich also der vermutete Zusammenhang zwischen der Beschäftigung vor und nach der Erwerbsminderungsrente.

Einen deutlichen Vorteil haben jene, die mit dem Ende der Erwerbsminderungsrente noch oder wieder beschäftigt waren: Unter ihnen ist der Anteil der Personen, die zwölf Monate nach Auslaufen der Erwerbsminderungsrente in Beschäftigung sind, mit 18,9\% fast doppelt so hoch wie unter jenen, die mit dem Ende der Erwerbsminderungsrente nicht beschäftigt waren. Die Kombination eines Beschäftigungsverhältnisses mit einer Erwerbsminderungsrente ist zum einen möglich bei 
einer Teilrente, kann zum anderen aber auch auf individuelle Vereinbarungen mit dem Arbeitgeber zurückgehen, was in den Daten jedoch nicht erkennbar ist. Dass der Anteil der Beschäftigten unter jenen, die bereits einer Beschäftigung am Ende der Erwerbsminderungsrente nachgingen, nach zwölf Monaten nicht höher ist als jene 18,9\%, liegt daran, dass die Mehrheit der hier erfassten Personen am Ende der Erwerbsminderungsrente das 60. Lebensjahr vollendet und deshalb aus der Analyse ausscheidet (siehe oben, Abschn. 4.1).

Im Durchschnitt lag das Entgelt in den fünf Jahren vor der Erwerbsminderungsrente bei 0,64 Entgeltpunkten aus einer Beschäftigung pro Monat, zwölf Monate nach der Erwerbsminderungsrente war es mit 0,69 Entgeltpunkten geringfügig höher. In beiden Fällen liegt das durchschnittliche Entgelt von Versicherten mit einer Erwerbsminderungsrentenepisode sowohl vor als auch nach der Rente also klar unter dem Durchschnitt aller Versicherten. Auch aus dieser Perspektive bestätigt sich, dass die gesundheitlichen Leistungseinschränkungen - die ja in der Regel vor und nach einer Erwerbsminderung bestehen, wenngleich in weniger gravierendem Ausmaß die Verdienstchancen beeinträchtigen.

Die Chance, nach einer Erwerbsminderungsrente wieder eine Beschäftigung zu finden, ist bei einem sehr niedrigen Verdienst vor der Erwerbsminderungsrente (0 bis 0,2 Entgeltpunkte) mit 9,5\% am niedrigsten. Wer in den fünf Jahren vor seiner Erwerbsminderungsrente mehr als 0,2 und bis zu 0,6 Entgeltpunkte erwarb, hatte mit $15 \%$ eine etwas höhere Wahrscheinlichkeit, zwölf Monate nach Ende der Erwerbsminderungsrente wieder in Beschäftigung zu sein. In den drei Gruppen mit einem Einkommen oberhalb von $60 \%$ des Durchschnittsverdienstes unterscheiden sich die Übergangswahrscheinlichkeiten in Beschäftigung mit Werten zwischen knapp 12 und knapp $13 \%$ kaum.

\subsection{Einflussfaktoren auf die Übergangswahrscheinlichkeit in Beschäftigung}

Im Folgenden wird durch eine logistische Regressionsrechnung geprüft, ob die deskriptiv gefundenen Zusammenhänge auch bei Kontrolle aller anderen bisher diskutierten Variablen Bestand haben. Hierzu wird auf Basis eines ungewichteten Regressionsmodells diskutiert, ${ }^{10}$ ob sich die Übergangswahrscheinlichkeiten zwischen unterschiedlichen Ausprägungen der erklärenden Variablen signifikant unterscheiden (vgl. Tab. 4).

Aus der ungewichteten Regression sind vor allem vier Ergebnisse hervorzuheben (siehe Zusammenfassung in Tab. 5). Erstens: Ältere ab 56 Jahren haben eine signifikant niedrigere Beschäftigungswahrscheinlichkeit als Jüngere bis 40 Jahre. Die mittlere Altersgruppe zwischen 41 und 55 Jahren unterscheidet sich hingegen nicht signifikant von den Jüngeren. Die Hypothese 2 zur Altersabhängigkeit der Beschäftigungschance kann somit bestätigt werden. Zweitens: Dauert eine Erwerbsminderungsrente länger als ein Jahr, ist die Beschäftigungschance signifikant höher

\footnotetext{
10 Nach Solon et al. (2013) ist eine ungewichtete Regression zu bevorzugen, wenn nicht konkrete Gründe für eine Gewichtung sprechen. Dies können etwa sein: Korrektur von Heteroskedastizität, Ausgleich für eine endogene Fallauswahl und Ermittlung eines gewichteten Effekts für die Gesamtpopulation. Diese Gründe sind hier nicht gegeben.
} 
Tab. 4 Logistische Regression ohne Gewichtung (Logit-Koeffizienten und Signifikanzniveaus). (Quelle: SUFVSKT 2015; eigene Berechnungen)

\begin{tabular}{|c|c|c|}
\hline Variable & Variablenausprägung & Logit-Koeffizient \\
\hline \multirow{3}{*}{$\begin{array}{l}\text { Geburtsjahr (gruppiert) } \\
\text { Ref. } 1957 / 1960\end{array}$} & $1948 / 1956$ & 0,48 \\
\hline & $1961 / 1969$ & $-0,01$ \\
\hline & $1970 / 1985$ & 0,18 \\
\hline \multirow{2}{*}{$\begin{array}{l}\text { Altersgruppe } \\
\text { Ref. } \leq 40\end{array}$} & $41-55$ & $-0,48$ \\
\hline & $\geq 56$ & $-3,51 * * *$ \\
\hline \multicolumn{3}{|l|}{$\begin{array}{l}\text { Geschlecht } \\
\text { Ref. Frauen }\end{array}$} \\
\hline \multirow{4}{*}{$\begin{array}{l}\text { Dauer der EM-Rente (gruppiert) } \\
\text { Ref. } \leq 1 \mathrm{~J} \text {. }\end{array}$} & $>1 \mathrm{~J}$. u. $\leq 3 \mathrm{~J}$ & $2,07 * * *$ \\
\hline & $>3 \mathrm{~J}$. u. $\leq 6 \mathrm{~J}$ & $1,64 * * *$ \\
\hline & $>6 \mathrm{~J}$. u. $\leq 9 \mathrm{~J}$ & $2,12 * * *$ \\
\hline & $>9 \mathrm{~J}$. & $1,82 * * *$ \\
\hline \multirow{4}{*}{$\begin{array}{l}\text { Entgelt in Relation zum Durchschnitt in den } 5 \text { Jahren } \\
\text { vor der EM-Rente } \\
\text { Ref. }>80 \% \text { u. } \leq 120 \%\end{array}$} & $0-20 \%$ & 0,89 \\
\hline & $>20 \%$ u. $\leq 60 \%$ & $-0,07$ \\
\hline & $>60 \%$ u. $\leq 80 \%$ & $-0,07$ \\
\hline & $>120 \%$ & 0,07 \\
\hline $\begin{array}{l}\text { Beschäftigung im letzten Monat der EM-Rente } \\
\text { Ref. keine Beschäftigung }\end{array}$ & Beschäftigung & $0,78^{* * *}$ \\
\hline \multirow{2}{*}{$\begin{array}{l}\text { Monate mit Beschäftigung in den } 5 \text { Jahren vor der } \\
\text { EM-Rente } \\
\text { Ref. keine Beschäftigung }\end{array}$} & 1 Monat bis $3 \mathrm{~J}$. & $2,16^{* * *}$ \\
\hline & $>3-5 \mathrm{~J}$ & $2,21 * * *$ \\
\hline \multirow{3}{*}{$\begin{array}{l}\text { Berufsausbildung } \\
\text { Ref. mit Berufsausbildung }\end{array}$} & ohne Berufsausbildung & 0,60 \\
\hline & Uni/FH & $1,85^{*}$ \\
\hline & Missing & $-1,29 * * *$ \\
\hline \multicolumn{2}{|l|}{ Modell-Konstante } & $-4,03 * * *$ \\
\hline \multicolumn{2}{|l|}{$N$} & 869 \\
\hline \multicolumn{2}{|l|}{ Pseudo- $\mathrm{R}^{2}$ (McFadden) } & 0,34 \\
\hline
\end{tabular}

Signifikanzniveaus: $* p<0,1 ; * * p<0,05 ; * * * p<0,01$

als bei kürzerer Rentendauer. Die Ausgangsvermutung und der zunächst deskriptiv gewonnene Befund, dass mit zunehmender Dauer der Erwerbsminderungsrente die Beschäftigungswahrscheinlichkeit zurückgeht (Hypothese 7), bestätigen sich daher nicht. Vielmehr scheint es eine länger andauernde Phase zu geben, innerhalb der sich erst klärt, ob die Erwerbsfähigkeit wieder erreicht werden kann. Drittens: Eine Beschäftigung vor der Erwerbsminderungsrente bringt höhere Beschäftigungschancen nach der Erwerbsminderungsrente mit sich als keine Beschäftigung vor der Erwerbsminderungsrente. Hypothese 6 wird im Grundsatz bestätigt. Damit einher geht, dass eine Beschäftigung im letzten Monat vor dem Ende einer Erwerbsminderungsrente die Beschäftigungswahrscheinlichkeit zwölf Monate nach deren Ende zwar nur geringfügig, aber signifikant anhebt. Bereits während der Erwerbsminderungsrente in einem Beschäftigungsverhältnis zu sein, verbessert demnach die Chancen auf ein nachhaltiges Beschäftigungsverhältnis, was Hypothese 8 bestätigt. Viertens gibt es eine schwach signifikante höhere Beschäftigungschance für Personen mit einer Hochschulbildung, wobei hier - wie in der Untersuchung insgesamt - offen bleibt, 
Tab. 5 Übersicht über die Ergebnisse zu den Hypothesen. (Quelle: Eigene Darstellung)

\begin{tabular}{lll}
\hline & Höhere Beschäftigungschance nach einer Erwerbsminderungsrente ... & Ergebnis \\
\hline H1 & bei Männern & $\begin{array}{l}\text { Kein signifikanter } \\
\text { Zusammenhang }\end{array}$ \\
H2 & mit niedrigerem Alter & Ja \\
H3 & $\begin{array}{l}\text { wenn nicht von der EM-Rentenreform 2001 betroffen (d.h. älter als } \\
\text { Jahrgang 1961) }\end{array}$ & Kein signifikanter \\
H4 & bei hoher oder niedriger Qualifikation (beide Richtungen denkbar) & Ja (bei hoher Qualifi- \\
& $\begin{array}{l}\text { bei hoher oder niedriger Einkommensposition vor der Erwerbsminde- } \\
\text { H5 }\end{array}$ & Kation) \\
H6 & weingsignifikanter \\
H7 & bei kürzerer Dauer der Erwerbsminderungsrente & Zusammenhang \\
H8 & wenn bereits vor Ende der Erwerbsminderungsrente beschäftigt & Ja \\
\hline
\end{tabular}

ob die alte Beschäftigung in angepasster Form fortgeführt werden konnte oder eine neue Beschäftigung gefunden wurde (Hypothese 4).

Aber auch die nicht signifikanten Befunde sind bemerkenswert. Sie widersprechen teilweise den Ausgangsvermutungen: So hat die Kohortenzugehörigkeit und damit die Betroffenheit von der Verschärfung des Erwerbsminderungsrentenrechts 2001 keine nachweisbaren Auswirkungen auf die Chance, nach dem Ende eines Erwerbsminderungsrentenbezugs in Beschäftigung zurückzukehren (Hypothese 3). ${ }^{11}$ Auch nach dem Geschlecht ist kein Unterschied in den Übergangswahrscheinlichkeiten in Beschäftigung zu erkennen (Hypothese 1). Weiterhin gibt es keinen klaren Zusammenhang zwischen der Entgeltposition vor der Erwerbsminderungsrente und der Beschäftigungschance danach (Hypothese 5).

Als Maßzahl für die Qualität der Gesamtschätzung wurde das modellspezifische Gütemaß Pseudo-R ${ }^{2}$ (McFadden) berechnet. Es kann Werte zwischen „Null“ und „Eins“ annehmen, ist aber kein exaktes Maß für die erklärte Varianz durch das spezifizierte Modell. In der vorliegenden Berechnung liegt der entsprechende Wert bei rund 0,34, was für eine gute Modellschätzung spricht (Urban 1995).

\section{Zusammenfassung und Diskussion}

Von den knapp 35 Mio. Rentenversicherten, die die Grundlage der vorliegenden Untersuchung bildeten, durchlebten knapp 1,9 Mio. irgendwann im Laufe ihres Erwerbslebens eine Phase der Erwerbsminderung. Die Analysen haben gezeigt, dass Phasen des Erwerbsminderungsrentenbezugs vielfach in instabile Erwerbsverläufe eingebettet sind. Dies ist für die Wege in Erwerbsminderung vielfältig dokumentiert (Brussig 2012; Gasche und Härtl 2013; Mittag et al. 2014; Söhn und Mika 2015, 2019). Die vorliegende Untersuchung konzentrierte sich auf Erwerbschancen nach dem Ende eines Erwerbsminderungsrentenbezugs, und dies vor dem Hintergrund

$11 \mathrm{Zu}$ vermuten ist aber, dass die Reform des Erwerbsminderungsrentenrechts die Häufigkeit der Befristung und die Zugangschancen in Erwerbsminderungsrenten beeinflusst hat. 
der Tatsache, dass seit der Reform des Erwerbsminderungsrechts 2001 Befristungen den Regelfall bilden sollen.

Die Instabilität der Erwerbsverläufe schon vor dem Bezug einer Erwerbsminderungsrente ist Ausdruck eines eingeschränkten Leistungsvermögens, das oft über einen längeren Zeitraum sinkt und bereits vor einer Erwerbsminderung mit Phasen der Nichtbeschäftigung einhergeht. Nach dem Auslaufen einer Erwerbsminderungsrente hält die Instabilität an. Das Ende des Bezugs einer Erwerbsminderungsrente zeigt im Regelfall also keine dauerhaft wiederhergestellte Fähigkeit an, einem Erwerb nachzugehen. Zwölf Monate nach dem Ende eines Erwerbsminderungsrentenbezugs sind 12,6\% der Betreffenden versicherungspflichtig oder (zu einem sehr geringen Anteil) geringfügig beschäftigt. Wiederum die Hälfte dieser Beschäftigten war bereits am Ende des Erwerbsminderungsrentenbezugs erwerbstätig; hier ist zu vermuten - was aber in den Daten nicht erkennbar ist -, dass es sich um eine Teilerwerbsminderung bzw. Teilerwerbstätigkeit handelte, die nach dem Auslaufen der Rente fortgeführt wurde. Unmittelbar nach dem Ende des Erwerbsminderungsrentenbezugs bzw. sechs Monate danach waren noch etwas mehr erwerbstätig (15,0 bzw. $14,6 \%$ ) als ein Jahr danach. Der Rückgang der Integrationsquoten ist Ergebnis eines Sortierprozesses, in dem bestehende Beschäftigungsverhältnisse häufiger aufgelöst als neue Beschäftigungsverhältnisse mit ehemals Erwerbsgeminderten eingegangen werden. Mit wachsender zeitlicher Entfernung vom Erwerbsminderungsrentenbezug stabilisieren sich die verbleibenden Beschäftigungsverhältnisse. Etwa die Hälfte der Erwerbsminderungsrenten endet aufgrund der in dieser Untersuchung zugrundegelegten Definition an der Altersgrenze von 60 Jahren. Für diese Personen war die Erwerbsminderungsrente faktisch dauerhaft, da sie jenseits des 60. Lebensjahres nahezu immer nahtlos in Altersrente führt.

Basierend auf dem Merkmalsspektrum des verwendeten Datensatzes, der Versichertenkontenstichprobe der Deutschen Rentenversicherung, konnten Unterschiede in der Häufigkeit der Erwerbsintegration gefunden werden, und es konnten auch Annahmen über die Gründe für diese Unterschiede zurückgewiesen werden. Insbesondere das Alter hängt mit der Rückkehrwahrscheinlichkeit zusammen: Jüngere Versicherte (unter 40 Jahre) kehren deutlich öfter in Beschäftigung zurück (36\%) als ältere Versicherte, denen es kaum gelingt (ca. 6\% der über 55-Jährigen). Fasst man Übergänge in Beschäftigung und Arbeitslosigkeit zusammen, gelingt mehr als der Hälfte der Jüngeren ein Übergang in einen arbeitsmarktnahen Zustand (ca. 55\%).

Eine hohe Beschäftigungsintensität vor der Erwerbsminderung erhöht die Chance, danach wieder in Beschäftigung zu kommen. Eine zunehmende Dauer einer Erwerbsminderungsrente geht nicht mit geringeren Beschäftigungschancen einher, wenn man den parallelen Effekt des Älterwerdens kontrolliert.

Keinen Unterschied gibt es in der Kohortenzugehörigkeit bzw. in der Betroffenheit von der Reform des Erwerbsminderungsrentenrechts 2001, mit der der Zugang zur Rente erschwert und die Leistungen abgesenkt wurden. Insoweit es Ziel der Reform war, durch ein ,aktivierendes“ institutionelles Design die Wahrscheinlichkeit einer Rückkehr in Beschäftigung zu erhöhen, liefern die Daten keinen Anhaltspunkt dafür, dass dies gelungen wäre.

Die hier vorgelegten Ergebnisse zur Rückkehr in Beschäftigung entsprechen Ergebnissen bereits vorliegender Studien (Köckerling et al. 2019; Lohse und Rodriguez 
Gonzalez 2015), gehen aber in der Differenzierung nach personalen und erwerbsbiografischen Merkmalen über sie hinaus. Dies ermöglicht genauere Einschätzungen zu der Frage, wem warum der Übergang in eine Beschäftigung nach dem Auslaufen einer befristeten Erwerbsminderungsrente gelingt. Gleichwohl bleibt eine Reihe von Fragen offen. Wünschenswert ist die Aufnahme von weiteren Informationen aus dem Scientific Use File, die grundsätzlich vorhanden sind, insbesondere zum Teilrentenbezug und zur Rentenart. Andere potenziell erklärungsstarke Informationen lassen sich nicht über das Meldeverfahren der Sozialversicherungen beschaffen, auf dem der genutzte Datensatz beruht. So ist zu vermuten, dass eine Partnerschaft und das Haushaltseinkommen die Integrationswahrscheinlichkeit nach einem Erwerbsminderungsrentenbezug ebenso beeinflussen wie die Situation beim Arbeitgeber. Hierfür kommen grundsätzlich Haushaltsbefragungen wie z. B. das SOEP oder Betriebsbefragungen wie z.B. das IAB-Betriebspanel infrage, allerdings werden weder im SOEP noch in den vorliegenden Betriebsbefragungen Fragen zur Erwerbsminderung in ausreichender Tiefe angesprochen. Für Fragen zu den beruflichen (Neu-)Orientierungen von Versicherten vor, während und nach einer Erwerbsminderung, die ebenfalls Aufschluss zu den Rückkehrchancen versprechen, sind eigenständige Erhebungen und eher sinnrekonstruktive Forschungsansätze nötig (siehe oben Abschn. 3, Zschucke et al. 2016 sowie Zschucke und Lippke 2017).

Lassen sich trotz der bestehenden Wissenslücken sozialpolitische Schlussfolgerungen aus der Untersuchung ziehen? Angesichts der Instabilität von Phasen der Erwerbsfähigkeit und der Arbeitsunfähigkeit sind Sicherungsmechanismen sinnvoll, die nicht einfach nur zwischen dauerhafter Erwerbsfähigkeit und dauerhafter Erwerbsminderung unterscheiden. Insofern scheint der Grundsatz einer befristeten Erwerbsminderungsrente prinzipiell angemessen. Auch die Tatsache, dass ein erheblicher Teil der Personen zwölf Monate nach Ende der befristeten Erwerbsminderung erneut eine Erwerbsminderungsrente bezieht, spricht nicht grundsätzlich gegen eine Befristung. Problematisch wäre es vielmehr, wenn ehemals Erwerbsgeminderte nicht erneut in eine Erwerbsminderungsrente zurückkehren könnten und wenn unter den ehemals Erwerbsgeminderten viele Personen wären, die nach wie vor faktisch erwerbsgemindert sind, ohne eine Rente beziehen zu können. Ob eine Integrationsquote von $12,6 \% \mathrm{zu}$ niedrig ist, um eine grundsätzliche Befristung der Erwerbsminderungsrente $\mathrm{zu}$ rechtfertigen, ist empirisch kaum $\mathrm{zu}$ entscheiden. Man wird aber sagen können, dass angesichts von 8,7\% ehemaliger Erwerbsminderungsrentner_innen, die zwölf Monate nach dem Erwerbsminderungsrentenbezug arbeitslos sind, noch ein beträchtliches Potenzial besteht, mittels Arbeitsförderung ihnen zu einer Beschäftigung zu verhelfen. Dieses Potenzial besteht insbesondere bei Jüngeren, aber kaum bei Älteren. Wie eine angemessene Arbeitsförderung aussehen könnte, ist Gegenstand von akademischen Diskussionen (vgl. statt vieler Knuth 2014) und praktischen Modellprogrammen, die insbesondere die Möglichkeiten öffentlich geförderter Beschäftigung (IAQ et al. 2019; ISG und IAW 2018) und einer verbesserten Zusammenarbeit von Jobcentern und der Rentenversicherung (Ahuja 2019) erproben und teilweise bereits regelhaft praktizieren (Kupka et al. 2019). Der Befund aus diesem Beitrag, dass es einen längeren Sortierprozess gibt, in dem sich erst im Laufe der Zeit herausstellt, ob leistungsgeminderte Personen auf leidensgerechte Arbeitsplätze gelangt sind, legt die Schlussfolgerung nahe, die ,aktivierende“ Er- 
werbsaufforderung mit angepassten Beschäftigungsangeboten und Rückkehrrechten in die Erwerbsminderungsrente bei fehlgeschlagenen Integrationsversuchen zu verbinden. Dies soll dazu beitragen, die Risikobereitschaft der betroffenen Personen zu stärken, um eine Beschäftigungsaufnahme trotz hoher Scheiternsrisiken zu wagen.

Neben einer Arbeitsförderung, die speziell auf die Belange von jüngeren Menschen mit eingeschränkter Leistungsfähigkeit zugeschnitten ist, erscheint es außerdem gerechtfertigt, über eine spezielle „Erwerbsminderungsrente für Ältere“ zu diskutieren (siehe auch Bäcker et al. 2011, S. 28f.; DGB 2007), die deren schlechteren Integrationschancen Rechnung trägt und beispielsweise auf die grundsätzliche Befristung verzichtet. Eine solche Weiterentwicklung der Erwerbsminderungsrente könnte dazu beitragen, die anhaltende Skepsis unter den Versicherten gegenüber der Anhebung der Altersgrenzen bei den Altersrenten zu dämpfen und das Vertrauen in die soziale Sicherung zu stärken.

Danksagung Wir bedanken uns bei den beiden anonymen Gutachter_innen des Beitrages für ihre konstruktiven Hinweise.

Funding Open Access funding provided by Projekt DEAL.

Open Access Dieser Artikel wird unter der Creative Commons Namensnennung 4.0 International Lizenz veröffentlicht, welche die Nutzung, Vervielfältigung, Bearbeitung, Verbreitung und Wiedergabe in jeglichem Medium und Format erlaubt, sofern Sie den/die ursprünglichen Autor(en) und die Quelle ordnungsgemäß nennen, einen Link zur Creative Commons Lizenz beifügen und angeben, ob Änderungen vorgenommen wurden.

Die in diesem Artikel enthaltenen Bilder und sonstiges Drittmaterial unterliegen ebenfalls der genannten Creative Commons Lizenz, sofern sich aus der Abbildungslegende nichts anderes ergibt. Sofern das betreffende Material nicht unter der genannten Creative Commons Lizenz steht und die betreffende Handlung nicht nach gesetzlichen Vorschriften erlaubt ist, ist für die oben aufgeführten Weiterverwendungen des Materials die Einwilligung des jeweiligen Rechteinhabers einzuholen.

Weitere Details zur Lizenz entnehmen Sie bitte der Lizenzinformation auf http://creativecommons.org/ licenses/by/4.0/deed.de.

\section{Literatur}

Ahuja, V. (2019). Das Bundesprogramm rehapro - Innovative Ideen für die Rehabilitation. Die Berufliche Rehabilitation, 33(1), 10-23.

Aurich, P. (2011). Activating the unemployed - Directions and divisions in Europe. European Journal of Social Security, 13, 294-316.

Aurich-Beerheide, P., Brussig, M., \& Schwarzkopf, M. (2018). Zugangssteuerung in Erwerbsminderungsrenten. HBS-Study, 377. Düsseldorf: Hans-Böckler-Stiftung.

Autorengruppe Bildungsberichterstattung (2018). Bildung in Deutschland 2018. Ein indikatorengestützter Bericht mit einer Analyse zu Wirkungen und Erträgen von Bildung. Bielefeld: wbv $\mathrm{Pu}-$ blikation. https://www.bildungsbericht.de/de/bildungsberichte-seit-2006/bildungsbericht-2018/pdfbildungsbericht-2018/bildungsbericht-2018.pdf. Zugegriffen: Aug. 2019.

Bäcker, G. (2012). Erwerbsminderungsrenten: Strukturen, Trends und aktuelle Probleme. Altersübergangs-Report 2012-03. Duisburg, Düsseldorf. http://www.iaq.uni-due.de/auem-report/2012/201203/auem2012-03.pdf. Zugegriffen: Aug. 2019.

Bäcker, G., Stapf-Finé, H., \& Kistler, E. (2011). Erwerbsminderungsrente. Reformnotwendigkeit und Reformoptionen. Bonn: Friedrich-Ebert-Stiftung.

Bauer, F., \& Sieglen, G. (2018). Individuell verfestigte Langzeitarbeitslosigkeit. Arbeit. Zeitschrift für Arbeitsforschung, Arbeitsgestaltung und Arbeitspolitik, 27, 87-107. 
Bauer, T. K., Beyer, F., Bredtmann, J., Otten, S., Piel, J., Sabisch, K., \& Stroka, M. (2016). Die Auswirkungen von Familienarbeit auf die Arbeitsmarktpartizipation, das (Alters-)Einkommen und die Gesundheit von Frauen. Eine empirische Analyse. RWI Materialien Heft 102. Essen: Rheinisch-Westfälisches Institut für Wirtschaftsforschung.

Behrens, J. (1994). Der Prozess der Invalidisierung - das demographische Ende eines historischen Bündnisses. In C. Behrend (Hrsg.), Frühinvalidität - ein Ventil des Arbeitsmarkts? Berufs- und Erwerbsunfähigkeitsrenten in der sozialpolitischen Diskussion (S. 105-135). Berlin: DZA.

Brettschneider, A. (2007). Jenseits von Leistung und Bedarf. Zur Systematisierung sozialpolitischer Gerechtigkeitsdiskurse. Zeitschrift für Sozialreform, 53, 365-389.

Brettschneider, A., \& Klammer, U. (2016). Lebenswege in die Altersarmut. Biografische Analysen und sozialpolitische Perspektiven. Berlin: Duncker \& Humblot.

Briest, J. (2018). Lebensqualität, Inanspruchnahme von Gesundheitsleistungen und Motivation zur Rückkehr ins Erwerbsleben bei zeitlich befristeten Erwerbsminderungsrentnern. In Deutsche Rentenversicherung Bund (Hrsg.), 27. Rehabilitationswissenschaftliches Kolloquium (S. 186-188). Tagungsband. Berlin: DRV.

Brussig, M. (2012). Erwerbsminderung und Arbeitsmarkt: Arbeitslosigkeit und regionale Unterschiede prägen Zugänge in Erwerbsminderungsrenten. Altersübergangs-Report 2012-04. Duisburg, Düsseldorf. https://www.iaq.uni-due.de/auem-report/2012/2012-04/auem2012-04.pdf. Zugegriffen: Aug. 2019.

Bundesagentur für Arbeit (2017). Blickpunkt Arbeitsmarkt - Situation schwerbehinderter Menschen. Nürnberg: BfA.

Bundessozialgericht. Urteil vom 09.05.2012, Aktenzeichen B 5R 68/11 Rd. Nr. 18.

Deutsche Rentenversicherung (2016). FDZ-Biografiedatensätze - VSKT/VVL. Benutzerhinweise zu den Verlaufsmerkmalen und Merkmalen der Rentenberechnung. Stand: 16.02.2016 http://www.fdz-rv.de/ FdzPortalWeb/getRessource.do?key=sufvsktvvl-benutzerhinweise.pdf. Zugegriffen: März 2019.

Deutsche Rentenversicherung (2017). FDZ-Biografiedatensatz für die Biografiedaten der Versicherten (VSKT) 2015. Stand: 25.01.2018. http://www.fdz-rv.de/FdzPortalWeb/getRessource.do?key=sufvskt 2015_cdpln.pdf. Zugegriffen: März 2019.

Deutsche Rentenversicherung (Hrsg.). (2014). Rechtliche Arbeitsanweisungen und Gesetzestexte der Regionalträger der Deutschen Rentenversicherung sowie der Deutschen Rentenversicherung Knappschaft-Bahn-See. Berlin.

Deutscher Gewerkschaftsbund (DGB) (2007). Vorschläge zu einem flexiblen Übergang in die Rente (Nachfolgeregelung Altersteilzeit). Beschluss des DGB-Bundesvorstands vom 4. September 2007, Berlin. Berlin.

Dieckhoff, M. (2011). The effect of unemployment on subsequent job quality in Europe: A comparative study of four countries. Acta Sociologica, 54, 233-249.

Eriksson, S., \& Lagerström, J. (2012). Detecting discrimination in the hiring process: Evidence from an Internet-based search channel. Empirical Economics, 43, 537-563.

Erlinghagen, M. (2006). Erstarrung, Beschleunigung oder Polarisierung. Arbeitsmarktmobilität und Beschäftigungsstabilität im Zeitverlauf. Neue Ergebnisse mit der IAB-Beschäftigtenstichprobe. Gelsenkirchen: Institut Arbeit und Technik.

Erlinghagen, M. (2008). Self-perceived job insecurity and social context: A multi-level analysis of 17 European countries. European Sociological Review, 24, 183-197.

Erlinghagen, M. (2017). Langfristige Trends der Arbeitsmarktmobilität, Beschäftigungsstabilität und Beschäftigungssicherheit in Deutschland. Duisburger Beiträge zur soziologischen Forschung 2017/05. Duisburg: Universität Duisburg Essen.

Erlinghagen, M., \& Knuth, M. (2010). Unemployment as an institutional construct? Structural differences in non-employment between selected European countries and the United States. Journal of Social Policy, 39, 71-94.

Fay, R. G. (1996). Enhancing the effectiveness of active labour market policies: Evidence from programme evaluations in OECD countries. Paris: OECD.

Fraktionen der SPD und Bündnis 90/Die Grünen (2000). Entwurf eines Gesetzes zur Reform der Renten wegen verminderter Erwerbsfähigkeit. BT-Drs. 14/4230.

Franz, C., Kümmerling, A., Lehndorff, S., \& Anxo, D. (2012). Arbeitszeiten von Frauen: Lebenslaufperspektive und europäische Trends. WSI-Mitteilungen, 65(8), S. 601-608.

Fuchsloch, C. (2011). Nahtlosigkeit und Einkommensersatz bei Leistungen. In K. J. Bieback, C. Fuchsloch, \& W. Kohte (Hrsg.), Arbeitsmarktpolitik und Sozialrecht (S. 135-148). München: Beck.

Gabriel, Y., Gray, D., \& Goregaokar, H. (2013). Job loss and its aftermath among managers and professionals: Wounded, fragmented and flexible. Work, Employment and Society, 27, 56-72. 
Gasche, M., \& Härtl, K. (2013). Verminderung der (Alters-)Armut von Erwerbsminderungsrentnern durch Verlängerung der Zurechnungszeit und Günstigerprüfung? MEA Discussion Papers 06-2013. München.

Hagen, C., \& Himmelreicher, R. (2014). Erwerbsminderungsrente in Deutschland - Ein unterschätztes Risiko(?). Vierteljahreshefte zur Wirtschaftsforschung, 83, 115-138.

Hagen, C., Himmelreicher, R., Kemptner, D., \& Lampert, T. (2011). Soziale Ungleichheit und Risiken der Erwerbsminderung. WSI-Mitteilungen, 64(7), 336-344.

Heinrich, R., Jochem, S., \& Siegel, N. A. (2016). Die Zukunft des Wohlfahrtsstaates. Einstellungen zur Reformpolitik in Deutschland. Bonn: Friedrich-Ebert-Stiftung. http://library.fes.de/pdf-files/wiso/ 12648.pdf. Zugegriffen: Aug. 2019.

Himmelreicher, R., \& Stegmann, M. (2008). New possibilities for socio-economic research through longitudinal data from the research data centre of the German Federal Pension Insurance (FDZ-RV). Journal of Contextual Economics - Schmollers Jahrbuch, 128(4), 647-660.

IAQ, ZEW, Zoom \& SOKO (2019). Evaluation des Bundesprogramms ,Soziale Teilhabe am Arbeitsmarkt". Endbericht. Berlin: Bundesministerium für Arbeit und Soziales.

ISG \& IAW (2018). Evaluation des ESF-Bundesprogramms zur Eingliederung langzeitarbeitsloser Leistungsberechtigter nach dem SGB II auf dem allgemeinen Arbeitsmarkt. Zwischenbericht. Berlin: Bundesministerium für Arbeit und Soziales.

Kirchner, S. (2016). Besser, schlechter oder polarisiert? Universelle und regime-spezifische Trends der Arbeitsqualität in der EU-15. Zeitschrift für Soziologie, 45, 73-90.

Knuth, M. (2014). Wo, bitte, ist hier der Ausgang? Perspektiven für gesundheitlich beeinträchtigte Arbeitslose. Bonn: Friedrich-Ebert-Stiftung.

Köckerling, E., Sauzet, O., Hesse, B., Körner, M., \& Razum, O. (2019). Return to Work aus einer zeitlich befristeten Erwerbsminderungsrente. Das Gesundheitswesen (eFirst). https://doi.org/10.1055/a0883-5276.

Krause, A., \& Köhler, C. (Hrsg.). (2012). Arbeit als Ware. Zur Theorie flexibler Arbeitsmärkte. Bielefeld: transcript.

Kümmerling, A., Postels, D., \& Slomka, C. (2015). Arbeitszeiten von Männern und Frauen - alles wie gehabt? Analysen zur Erwerbsbeteiligung in Ost- und Westdeutschland. IAQ-Report 2015-02. Duisburg: IAQ.

Kupka, P., Ramos Lobato, P., \& Wolff, J. (2019). Die Einführung eines Sozialen Arbeitsmarktes für Langzeitarbeitslose war ein wichtiger Schritt. IAB-Forum, 27.09.2019. https://www.iab-forum.de/ die-einfuehrung-eines-sozialen-arbeitsmarktes-fuer-langzeitarbeitslose-war-ein-wichtiger-schritt/. Zugegriffen: Sept. 2019.

Lessenich, S. (2008). Die Neuerfindung des Sozialen. Der Sozialstaat im flexiblen Kapitalismus. Bielefeld: transcript.

Lietzmann, T., Kupka, P., Ramos Lobato, P., Trappmann, M., \& Wolff, J. (2018). Sozialer Arbeitsmarkt für Langzeitarbeitslose: Wer für eine Förderung infrage kommt. IAB-Kurzbericht 20/2018. Nürnberg: Institut für Arbeitsmarkt- und Berufsforschung.

Lohse, R., \& Rodriguez Gonzalez, M. (2015). Sozioökonomische Risikofaktoren und Erwerbsbiografien im Kontext der Erwerbsminderung. 12. Jahrestagung des FDZ-RV. Berlin: FDZ-RV.

Lott, Y. (2017). Flexible Arbeitszeiten: Eine Gerechtigkeitsfrage? Forschungsförderung Report, Nr. 1. Düsseldorf: Hans-Böckler-Stiftung.

Martin, J. (1998). What works among active labour market policies. Evidence from OECD countries' experiences. Occasional Papers 35. Paris: OECD.

Märtin, S., \& Zollmann, P. (2011). Sozioökonomische Situation von Personen mit Erwerbsminderung. Ergebnisse aus der Pilotstudie des Projektes der Deutschen Rentenversicherung Bund. RVaktuell, 58(4), 121-126.

Märtin, S., Zollmann, P., \& Buschmann-Steinhage, R. (2012). Sozioökonomische Situation von Personen mit Erwerbsminderung. Projektbericht I zur Studie. Berlin: DRV.

Märtin, S., Zollmann, P., \& Buschmann-Steinhage, R. (2015). Sozioökonomische Situation von Personen mit Erwerbsminderung. Projektbericht II zur Studie. Berlin: DRV.

Mika, T. (2013). Risiken für eine Erwerbsminderung bei unterschiedlichen Berufsgruppen. Bundesgesundheitsblatt - Gesundheitsforschung - Gesundheitsschutz, 56(3), 391-398.

Mika, T., Lange, J., \& Stegmann, M. (2014). Erwerbsminderungsrenten nach Bezug von ALG II: Auswirkungen der Reformen auf die Versicherungsbiographien. WSI-Mitteilungen, 67(4), 277-285.

Mittag, O., Reese, C., \& Meffert, C. (2014). (Keine) Reha vor Rente: Analyse der Zugänge zur Erwerbsminderungsrente 2005-2009. WSI-Mitteilungen, 67(2), 149-155. 
Müller, R., Hagen, C., \& Himmelreicher, R. (2013). Risiken für eine Erwerbsminderungsrente - Bremen im Ländervergleich. Bremen: Arbeitnehmerkammer Bremen.

Nüchter, O., Bieräugel, R., Schippers, F., Glatzer, W., \& Schmid, A. (2008). Einstellungen zum Sozialstaat II: Akzeptanz der sozialen Sicherung und der Reform der Renten- und Pflegeversicherung 2006. Opladen: Verlag Barbara Budrich.

OECD (1996). The OECD jobs strategy. Enhancing the effectiveness of active labour market policies. Paris: OECD.

OECD (2003). OECD employment outlook 2003. Towards more and better jobs. Paris: OECD.

OECD (2010). Sickness, disability and work. Breaking the barriers: A synthesis of findings across OECD countries. Paris: OECD.

Offe, C., \& Hinrichs, K. (1977). Sozialökonomie des Arbeitsmarktes und die Lage „,benachteiligter“ Gruppen von Arbeitnehmern. In C. Offe (Hrsg.), Opfer des Arbeitsmarktes. Zur Theorie der strukturierten Arbeitslosigkeit (S. 3-61). Neuwied: Luchterhand.

Paul, K., \& Moser, K. (2019). Ursachen und Auswirkungen von Arbeitslosigkeit. In S. Kauffeld \& D. Spurk (Hrsg.), Handbuch Karriere und Laufbahnmanagement (S. 655-685). Berlin: Springer.

Prinzen, K. (2016). Attitudes toward intergenerational redistribution in the welfare state. In K. Hank \& M. Kreyenfeld (Hrsg.), Social Demography. Forschung an der Schnittstelle von Soziologie und Demografie. Kölner Zeitschrift für Soziologie und Sozialpsychologie, Sonderheft 55 (S. 349-370). Wiesbaden: Springer VS.

Riphahn, R. (1997). Disability retirement and unemployment - substitute pathways for labour force exit? An empirical test for the case of Germany. Applied Economics, 29, 551-561.

Rodriguez Gonzalez, M., Lohse, R., Schröder, M., Krohn, S., \& Zuchandke, A. (2015). Sozioökonomische Analyse des Erwerbsminderungsrisikos - Eine Untersuchung anhand von BASiD-Daten. Zeitschrift für die gesamte Versicherungswissenschaft, 104, 151-178.

Ruland, F. (1990). Das Recht der Berufs- und Erwerbsunfähigkeitsrenten. Probleme und Reformmöglichkeiten. Zeitschrift für Sozialreform, 36, 486-501.

Ruland, F., \& Rische, H. (1980). Die „Erwerbsminderungsrente“ als Möglichkeit zur Reform der Sicherung bei Berufs- und Erwerbsunfähigkeit. Deutsche Rentenversicherung, 35(1), 12-29.

Schmähl, W. (2011). Die Entwicklung der Rentenversicherung vom Ende des Zweiten Weltkrieges bis zum Mauerfall (1945-1989). In E. Eichenhofer, H. Rische \& W. Schmähl (Hrsg.), Handbuch der Gesetzlichen Rentenversicherung, SGB VI (S. 33-66). Köln: Hermann Luchterhand Verlag.

Schneider, L. (2007). Zu alt für einen Wechsel? Zum Zusammenhang von Alter, Lohndifferentialen und betrieblicher Mobilität. Sozialer Fortschritt, 56(7/8), 180-186.

Schrenker, M. (2009). Warum fast alle das deutsche Rentensystem ungerecht finden, aber trotzdem nichts daran ändern möchten. Kölner Zeitschrift für Soziologie und Sozialpsychologie, 61, 259-282.

Schubert, M., Behrens, J., Höhne, A., Schaepe, C., \& Zimmermann, M. (2006). Erwerbsminderungsrenten wegen verschlossenem Arbeitsmarkt - der Arbeitsmarkt als Frühberentungsgrund. In Deutsche Rentenversicherung Bund (Hrsg.), Erfahrungen und Perspektiven. Bericht vom dritten Workshop des Forschungsdatenzentrums der Rentenversicherung (FDZ-RV) vom 26. bis 28. Juni 2006 in Bensheim. DRV-Schriften, 55 (S. 237-256). Bad Homburg: wbv.

Söhn, J., \& Mika, T. (2015). Die erwerbsbiografische Vorgeschichte der Frühverrentung wegen Erwerbsminderung. Zeitschrift für Sozialreform, 61, 461-492.

Söhn, J., \& Mika, T. (2019). Veränderung der Versicherungsbiografien im Rentenzugang 2003 bis $2014 \mathrm{im}$ Vergleich. Deutsche Rentenversicherung, 74(3), 259-288.

Solga, H. (2014). Education, economic inequality and the promises of the social investment state. SocioEconomic Review, 12, 269-297.

Solon, G., Haider, S. J., \& Wooldridge, J. (2013). What are we weighting for? NBER Working Paper 18859. Cambridge: National Bureau of Economic Research.

Stegmann, M. (2016). Methodische Umsetzung des SK79 in einen anonymisierten Datensatz fester Satzlänge: Sequentielle Biografiedaten. Würzburg: Forschungsdatenzentrum Deutsche Rentenversicherung.

Stone, D. (1985). The disabled state. London: Macmillan.

Urban, D. (1995). Logit-Analyse. Statistische Verfahren zur Analyse von Modellen mit qualitativen Response-Variablen. Stuttgart: Gustav Fischer.

Wuppinger, J., \& Rauch, A. (2010). Wiedereingliederung in den Arbeitsmarkt im Rahmen beruflicher Rehabilitation. Maßnahmeteilnahme, Beschäftigungschancen und Arbeitslosigkeitsrisiko. IAB-Forschungsbericht, 1/2010. Nürnberg: IAB.

Zschucke, E., \& Lippke, S. (2017). Erwerbsminderungsrente und Rückkehr ins Erwerbsleben aus Sicht der Betroffenen. Diskussionsforum Rehabilitations- und Teilhaberecht, Fachbeitrag D15-2017 unter www.reha-recht.de. Zugegriffen: Aug. 2019. 
Zschucke, E., Hessel, A., \& Lippke, S. (2016). Befristete Erwerbsminderungsrente aus Sicht der Betroffenen: subjektiver Gesundheitszustand, Rehabilitationserfahrungen und Pläne zur Rückkehr ins Erwerbsleben. Die Rehabilitation, 55, 223-229.

Martin Brussig geb. 1967. Prof. Dr., Soziologe, Leiter der Forschungsabteilung „Arbeitsmarkt - Integration - Mobilität“ am Institut Arbeit und Qualifikation (IAQ), Universität Duisburg-Essen. Forschungsschwerpunkte: Arbeitsmarktpolitik, Altersübergänge. Aktuelle Veröffentlichungen: (mit P. Aurich-Beerheide und M. Schwarzkopf) Zugangssteuerung in Erwerbsminderungsrenten, 2018; Was kommt nach der Aktivierung? Neue Leitbilder der Arbeitsmarktpolitik, in: Arbeit, 2019.

Susanne Eva Drescher geb. 1986. Dr., wissenschaftliche Mitarbeiterin am Institut Arbeit und Qualifikation (IAQ), Universität Duisburg-Essen. Forschungsschwerpunkte: Alterserwerbstätigkeit, Arbeitsmarktund Rentenpolitik. Aktuelle Veröffentlichungen: (mit M. Brussig) Soziale Unterschiede im Mortalitätsrisiko: Das frühere Arbeitsleben beeinflusst die fernere Lebenserwartung, 2019; (mit S. Stöbe-Blossey, M. Ruth und M. Brussig) Der komplexe Sozialstaat: Eine Heuristik zur Rekonstruktion von Schnittstellen, in: Sozialer Fortschritt, 2019.

Thorsten Kalina geb. 1971. Dr. rer. pol., Sozialwissenschaftler und wissenschaftlicher Mitarbeiter in der Forschungsabteilung „Flexibilität und Sicherheit“ am Institut Arbeit und Qualifikation (IAQ), Universität Duisburg-Essen. Mitarbeit in einer internationalen Vergleichsstudie zur Niedriglohnbeschäftigung in Europa und einer Studie zur Evaluation des Mindestlohns in der Gebäudereinigung in Deutschland. Forschungsschwerpunkte: Niedriglohnbeschäftigung, einfache Tätigkeiten und Entwicklung der Mittelschicht in Deutschland. 